\title{
Spatially resolved signature of quenching in star-forming galaxies
}

\author{
Salvatore Quai, ${ }^{1,2 \star}$ Lucia Pozzetti, ${ }^{2}$ Michele Moresco, ${ }^{1,2}$ Annalisa Citro, ${ }^{3}$ Andrea Cimatti \\ Jarle Brinchmann ${ }^{5,6}$, Madusha L. P. Gunawardhana ${ }^{6}$ and Mieke Paalvast ${ }^{6}$ \\ ${ }^{1}$ Dipartimento di Fisica e Astronomia, Università di Bologna, Via Gobetti 93/2, I-40129, Bologna, Italy \\ ${ }^{2}$ INAF - Osservatorio di Astrofisica e Scienza dello Spazio di Bologna, Via Gobetti 93/3, I-40129, Bologna, Italy \\ 3 The Leonard E. Parker Center for Gravitation, Cosmology and Astrophysics, Department of Physics, University of Wisconsin-Milwaukee, 3135 \\ ${ }^{4}$ INAF - Osservatorio Astrofisico di Arcetri, Largo E. Fermi 5, I-50125, Firenze, Italy \\ ${ }^{5}$ Instituto de Astrofisica e Ciências do Espaço, Universidade do Porto, CAUP, Rua das Estrelas, PT4150-762 Porto, Portugal \\ ${ }^{6}$ Leiden Observatory, Leiden University, PO Box 9513, 2300 RA, Leiden, The Netherlands
}

Accepted XXX. Received YYY; in original form ZZZ

\begin{abstract}
Understanding when, how and where star formation ceased (quenching) within galaxies is still a critical subject in galaxy evolution studies. Taking advantage of the new methodology developed by Quai et al. (2018) to select recently quenched galaxies, we explored the spatial information provided by IFU data to get critical insights on this process. In particular, we analyse 10 SDSS-IV MaNGA galaxies that show regions with low $[\mathrm{O} \mathrm{III}] / \mathrm{H} \alpha$ compatible with a recent quenching of the star formation. We compare the properties of these 10 galaxies with those of a control sample of 8 MaNGA galaxies with ongoing star formation in the same stellar mass, redshift and gas-phase metallicity range. The quenching regions found are located between 0.5 and 1.1 effective radii from the centre. This result is supported by the analysis of the average radial profile of the ionisation parameter, which reaches a minimum at the same radii, while the one of the star-forming sample shows an almost flat trend. These quenching regions occupy a total area between $\sim 15 \%$ and $45 \%$ of our galaxies. Moreover, the average radial profile of the star formation rate surface density of our sample is lower and flatter than that of the control sample, at any radii, suggesting a systematic suppression of the star formation in the inner part of our galaxies. Finally, the radial profile of gas-phase metallicity of the two samples have a similar slope and normalisation. Our results cannot be ascribed to a difference in the intrinsic properties of the analysed galaxies, suggesting a quenching scenario more complicated than a simple inside-out quenching.
\end{abstract}

Key words: galaxies: general - galaxies: evolution - galaxies: ISM

\section{INTRODUCTION}

Galaxies have pronounced bimodal distributions of their main properties (e.g. Strateva et al. 2001; Kauffmann et al. 2003; Blanton et al. 2003; Hogg et al. 2003; Balogh et al. 2004; Baldry et al. 2004; Bell et al. 2012). At higher redshifts, this bimodality has been confirmed up to $\mathrm{z} \sim 2$ (e.g. Willmer et al. 2006; Cucciati et al. 2006; Cirasuolo et al. 2007; Cassata et al. 2008; Kriek et al. 2008; Williams et al. 2009; Brammer et al. 2009; Muzzin et al. 2013). Moreover, there are strong evidences of a continuous growth, both in number density and stellar mass, of the red and passively

^ E-mail: salvatore.quai@unibo.it evolving early-type population from $\mathrm{z} \sim 1-2$ to the present (e.g. Bell et al. 2004; Blanton 2006; Bundy et al. 2006; Faber et al. 2007; Mortlock et al. 2011; Ilbert et al. 2013; Moustakas et al. 2013), suggesting that a large fraction of late-type galaxies transforms into early-type ones, as a consequence of the suppression of the star formation, together with a change in morphologies (e.g. Pozzetti et al. 2010; Peng et al. 2010). These transitional scenarios is thought to be dependent on the environment where galaxies are located (e.g. Goto et al. 2003; Balogh et al. 2004; Bolzonella et al. 2010; Peng et al. 2010). However, understanding when and how the star formation ceases (the so-called star formation quenching) and where it starts and propagates within star-forming galaxies is still one of the key open questions of galaxy evolution. 
The formation and evolution of disc galaxies in a hierarchical Universe (Fall \& Efstathiou 1980) leads to a scenario in which the outskirts of disc galaxies should form later than the inner part, by acquiring gas at higher angular momenta from the surrounding corona (the so-called inside-out growth Larson 1976). Inside-out growth is also predicted by hydrodynamical simulations (e.g. Pichon et al. 2011; Stewart et al. 2013) and it is supported by chemical evolution models (e.g. Boissier \& Prantzos 1999; Chiappini et al. 2001). This scenario is in agreement with numbers of observational evidences (e.g. Prantzos \& Boissier 2000; Gogarten et al. 2010; Spindler et al. 2018). Indeed, a natural consequence of inside-out growth is that central regions of galactic discs are, on average, older and more metal-rich than the outskirts (e.g. Zaritsky et al. 1994; Rosales-Ortega et al. 2011; Sánchez-Blázquez et al. 2014; González Delgado et al. 2014, 2015, 2016; Goddard et al. 2017a,b). This almost ubiquitous behaviour can be explained by a common evolution of gas, chemical history and stars (Ho et al. 2015), bearing in mind that without a continuous replenishing of fresh gas, galaxies would have fuel to sustain at most $\sim 1$ Gyr of star formation (Tacconi et al. 2013). In other words, the starforming galaxies need for a systematic supply of new gas and, together with evidence that inside-out growth is still active in outer part of most local star-forming galaxies (e.g. Wang et al. 2011; Muñoz-Mateos et al. 2011; Pezzulli et al. 2015), it suggests that galactic halos are still providing high angular momentum gas to assemble the out-skirts of galaxies. Moreover, starting from the evidence that hot coronae must rotate more slowly than the disc (i.e. pressure gradients provide support against gravity) Pezzulli \& Fraternali (2016) discussed that a misalignment between disc and halo velocity implies a systematic radial gas flow towards the inner parts of galaxies. Taking into account this effect and disentangling it from the contribution of inside-out growth in their models, these flows show a strong impact on the structural and chemical evolution of galaxies, naturally creating strong steep abundance gradient.

In this scenario, which mechanism drives the quenching of the star formation and how it can prevent further inflow of gas? Observational evidences of a systematic suppression of the star formation in the inner part of galaxies below the star-forming main sequence has been interpreted as an inside-out quenching (e.g. Tacchella et al. 2015; Belfiore et al. 2018; Ellison et al. 2018; Morselli et al. 2018; Lin et al. 2019). Being linked to AGN activities and gas outflows, they have suggested the negative AGN feedback as the mechanism that can trigger the interruption of the star formation from the centre and then, towards the outskirts. However, Tacchella et al. (2016) and recently Matthee \& Schaye (2019) and Wang et al. (2019) argued that the evidence of symmetry around the star-forming main sequence in the SFR - stellar mass diagram suggests an evolution of galaxies through phases of elevation and suppression of the star formation, without the need for a permanent quenching. This phenomenon is more clear in the inner part of galaxies because of the higher star formation efficiency, since higher gas fractions and shorter depletion times implicate shorter reaction time to the change in the reservoir of gas.

Therefore, identifying actual quenching galaxies that are leaving the blue cloud to reach the red sequence is still challenging. Having intermediate colours between blue late- type and red early-type galaxies, the so-called 'green valley' galaxies (Wyder et al. 2007; Martin et al. 2007; Salim et al. 2007; Schiminovich et al. 2007; Mendel et al. 2013; Salim 2014) have been considered as promising candidate for the transiting population. Schawinski et al. (2014), instead argued that green valley galaxies are actually separable into two populations of galaxies that share the same intermediate colours: (i) the green tail of the blue late-type galaxies with low specific star-formation rate but no sign of rapid transition towards early-type (quenching timescale of several Gyr) and (ii) a population of migrating early-type galaxies which are evolving (with timescale $\sim 1$ Gyr) to red and passive galaxies, as a result of major mergers of late-type galaxies. Belfiore et al. (2017a, 2018) recently found that the ionised optical spectra of most green valley galaxies are dominated by central low-ionization emission-lines (cLIER) due to old post-AGB stars radiation. The uniformity of old stellar populations suggests that green valley galaxies can be a 'quasistatic' population subjected to a slow-quenching. However, to account for the rate of growth of the red population and for the exiguity of transiting galaxies, there should be found galaxy populations in which star formation quenches on short timescales (e.g. Tinker et al. 2010; Salim 2014). Several hypothesis have been proposed to settle this puzzle. Some typical examples of galaxies quickly transforming into passively evolving galaxies are (i) galaxies which show both disturbed morphologies and intermediate colours (e.g. Schweizer \& Seitzer 1992; Tal et al. 2009) or (ii) strong morphological disturbances due to recent mergers (Hibbard \& van Gorkom 1996; Rothberg \& Joseph 2004; Carpineti et al. 2012), (iii) young elliptical galaxies (Sanders et al. 1988; Genzel et al. 2001; Dasyra et al. 2006) that are often characterized by low-level of recent star-formation (Kaviraj 2010) represent examples of galaxies that are quickly transforming into passively evolving galaxies. Studies regarding the so-called 'post-starburst' systems attempted to link the evolution of transient population with the properties of local early-type galaxies. This population shows strong Balmer absorption lines ( $\mathrm{H} \delta$ with equivalent width $>5 \AA$, in particular), typical of stellar populations dominated by A type stars with ages between $300 \mathrm{Myr}$ and 1 Gyr after the interruption of the star-formation (e.g. Couch \& Sharples 1987). Some of them have spectra compatible with passive evolution and no sign of emission lines (e.g. Quintero et al. 2004; Poggianti et al. 2004; Balogh et al. 2011; Muzzin et al. 2012; Mok et al. 2013; Wu et al. 2014) while others show emission lines (i.e. usually strong [O II] $\lambda 3726-29$ emission) and are often called 'strong-H $\delta$ ' galaxies (e.g. Le Borgne et al. 2006; Wild et al. $2009,2016)$. The properties of these galaxies are interpreted as sign of a recent fast-quenching (Dressler \& Gunn 1983; Zabludoff et al. 1996; Quintero et al. 2004; Poggianti et al. 2008; Wild et al. 2009). As a matter of fact, all these previous studies focused on galaxies observed 0.3-1 Gyr after the quenching phase. This delay, therefore, prevents to clearly unveil which processes drive the radical change in galaxy properties.

If, on one hand, stellar mass and metallicity are tracers of secular evolution of galaxies, on the other hand it is well known that the ionisation parameter (hereafter $\mathrm{U}$ ) provides powerful constraints on the recent activity within galaxies (e.g. Dopita et al. 2000; Kewley et al. 2001; Dopita et al. 2006; Levesque et al. 2010; Kewley et al. 2013; 
Kashino et al. 2016). Variations in the UV radiation strongly affect the galactic spectra. For example, the NUV continuum light, which it is primarily produced in the photosphere of long-lived stars more massive than $3 \mathrm{M}_{\odot}$, can trace the star formation on a timescale of $\sim 100 \mathrm{Myr}$. The Balmer lines, instead, are generated from the recombination of Hydrogen ionised by photons with energy higher than $912 \AA$ and only stars more massive than late-B stars irradiate a sufficient amount of UV flux to do this task. Thus, the $\mathrm{H} \alpha$ luminosity can trace SFR over the lifetime of these stars of tens of Myr. Spectral lines such as [O III] $\lambda 5007$ and [Ne III] $\lambda 3869$ can instead be produced only by the even more energetic photons coming from the short-lived, super massive $\mathrm{O}$ and early B stars. Therefore, these spectral lines are expected to disappear from galaxy spectra on timescales of 10-80 Myr, which correspond to the lifetime of the most massive stars, once that the SF stops. Citro et al. (2017, hereafter C17) and Quai et al. (2018, hereafter Q18) developed an innovative approach which aims at finding galaxies immediately after the quenching. The method is bases on the use of ratios between high-ionization potential lines (which can be produced only by very high energetic photons) such as [O III] and [Ne III], and low-ionization potential lines (which require lower energy photons) such as $\mathrm{H} \alpha, \mathrm{H} \beta,[\mathrm{OII}]$. C17 proved that $[\mathrm{O} \mathrm{III}] / \mathrm{H} \alpha$ ratio is a very sensitive tracer of the ongoing quenching as it drops by a factor $\sim 10$ within $\sim 10 \mathrm{Myr}$ from the quenching assuming a sharp interruption of the star formation, and even for a smoother and slower star formation decline (i.e. an exponential declining star formation history with $e$-folding time $\tau=200 \mathrm{Myr}$ ) the [O III] $/ \mathrm{H} \alpha$ decreases by a factor $\sim 2$ within $\sim 80 \mathrm{Myr}$ from the quenching. The $[\mathrm{O}$ III $] / \mathrm{H} \alpha$ ratio is affected by a significant degeneracy between ionization and metallicity (herafter Z), in the sense that $[\mathrm{O}$ III $] \lambda 5007$ emission can be depressed also by high metallicity (U-Z degeneracy, hereafter). In Q18, we found that the U-Z degeneracy can be mitigated by using couples of emission line ratios orthogonally dependent on ionisation (i.e. $[\mathrm{O} \mathrm{III}] / \mathrm{H} \alpha$ ) and metallicity (e.g. $[\mathrm{N} \mathrm{II}] /[\mathrm{O} \mathrm{II}]$ is a good tracer of gas-phase metallicity, as discussed in Kewley \& Dopita 2002; Nagao et al. 2006). In Q18 we used the $[\mathrm{O}$ III $] / \mathrm{H} \alpha$ vs. [N II] /[O II] diagnostic diagram in the SDSS to identify a sample of candidates quenching galaxies (QGs), i.e. in the early phase of quenching star formation, as a population well segregated from the global sample of galaxies with ongoing star-formation, showing $[\mathrm{O} \mathrm{III}] / \mathrm{H} \alpha$ ratios, at fixed $[\mathrm{NII}] /[\mathrm{O} \mathrm{II}]$, so low that they cannot be explained by metallicity effects.

Since the advent of integral field unit (IFU) spectroscopy era, galaxies can be studied with enough spatial resolution to allow analysis of physical properties even at galactocentric distances larger than 2 effective radii. In this paper, we extend the method devised in Q18 to select quenching galaxies in the SDSS main sample to IFU data from the SDSS-IV MaNGA survey (Bundy et al. 2015; Blanton et al. 2017). Our aim is to search for regions where quenching had started and, therefore, to derive spatial information on the quenching process within galaxies. This paper is intended to be a pilot study, where we analyse the more promising galaxies starting from the sample of SDSS QGs previously analyzed by Q18. We already planned to extend this study to the whole MaNGA population of star forming objects to

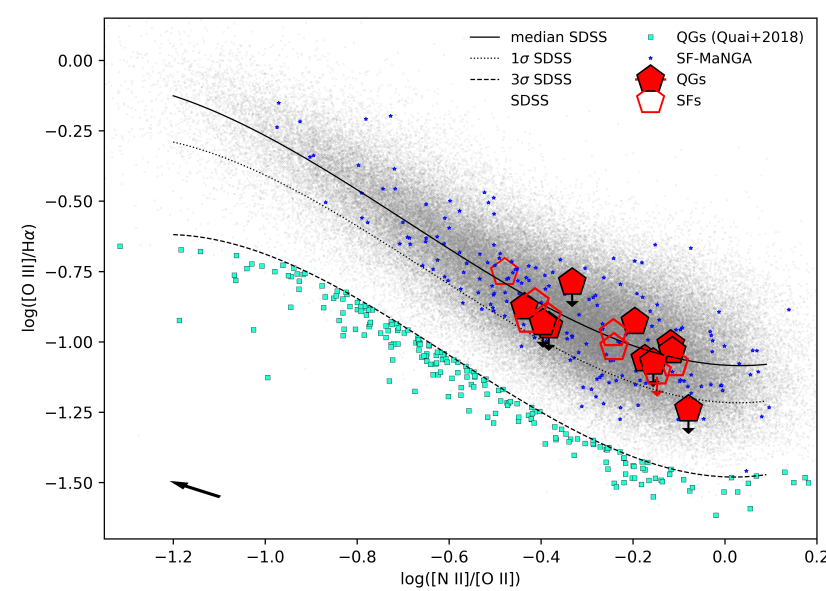

Figure 1. The diagnostic $[\mathrm{O} I \mathrm{II}] / \mathrm{H} \alpha$ vs $[\mathrm{N}$ II] $/[\mathrm{O}$ II] diagram. The black curves represent median, $1 \sigma$ and $3 \times 1 \sigma$ limits of the SDSS star-forming galaxies sample (see Q18), which are represented by grey dots. The black arrow in the bottom-left corner represents the direction of the dust vectors for the Calzetti et al. (2000) extinction law, for an $\mathrm{E}(\mathrm{B}-\mathrm{V})=0.3$. The cyan squared dots below the $3 \times 1 \sigma$ limits represent the SDSS quenching candidates selected in Q18. The blue dots represent the SDSS galaxies that have a match in MaNGA-DR14. The red pentagons represent the SDSS position of the MaNGA galaxies analysed in this paper: full symbols for the galaxies with quenching regions (QRG) and empty symbols for the star forming (SF) galaxies, as defined in subsection 2.5. The arrows indicate galaxies with the upper limits in $[\mathrm{O} \mathrm{III}] / \mathrm{H} \alpha$.

derive the total fraction of galaxies partially quenching and their properties.

We structure this paper as follows: in section 2 we briefly recall the method introduced in Q18 and we describe our MaNGA sample. We use section 3 to focus on two cases illustrating the detailed procedure and analysis done and then, in section 4 we present the general properties of the entire sample. Finally, in section 5 we discuss our results and we provide our concluding remarks.

\section{METHOD AND SAMPLE}

In Q18, from the analysis of a sample of $\sim 174.000$ starforming galaxies at $0.04<\mathrm{z}<0.21$ extracted from the SDSSDR8 catalogue, using the devised $[\mathrm{O}$ III] $/ \mathrm{H} \alpha$ vs. [N II] $/[\mathrm{O}$ II] diagnostic diagram, we identified about 300 quenching galaxy candidates satisfying the following criteria:

(i) [O III] weak enough to be undetected inside the SDSS fibre (i.e. $\mathrm{S} / \mathrm{N}([\mathrm{O}$ III $])<2$ ),

(ii) $[\mathrm{O}$ III $] / \mathrm{H} \alpha$ ratios, at fixed $[\mathrm{N} \mathrm{II}] /[\mathrm{O}$ II $]$ (i.e. fixed gasphase metallicity), lower than the $3 \times 1 \sigma$ value of the SDSS star-forming distribution (see Figure 1). They represent a population of galaxies well segregated from the global sample of galaxies with ongoing star-formation.

In order to derive spatial information on the quenching process within the galaxies, we extend these criteria to MaNGA IFU observations by exploiting the $[\mathrm{O}$ III $] / \mathrm{H} \alpha$ vs $[\mathrm{N}$ II $] /[\mathrm{O}$ II $]$ diagnostic of spatially resolved galaxies. To this aim, we cross-match the $\sim 174.000$ galaxies selected in the main 
SDSS survey (Q18) with the MaNGA data-release 14 (Abolfathi et al. 2018), finding 208 matches. However, none of 300 SDSS quenching primary candidates selected in Q18 has been observed with MaNGA. Nevertheless, we find matches with MaNGA data for 10 galaxies, among 26000 galaxies with $[\mathrm{O}$ III $]$ undetected $(\mathrm{S} / \mathrm{N}([\mathrm{O}$ III $])<2)$ within the SDSS fibre, which should represent promising candidates of galaxies which could be in the very first phase of the quenching. In fact, in Q18 we performed a survival analysis (ASURV, i.e. Kaplan-Meier estimator) of their $[\mathrm{O}$ III $] / \mathrm{H} \alpha$ distribution in slices of $[\mathrm{NII}] /[\mathrm{O} \mathrm{II}]$, and found that about $50 \%(3 \%)$ of them (that we called [O III] undet galaxies) are statistically distributed below $1 \sigma(3 \sigma)$ curve, respectively, and therefore candidates quenching galaxies, while the other ones should actually be normal star-forming galaxies with fainter emission lines. Among the 10 MANGA matches galaxies we discard MaNGA 1-245686 because it appears almost edge-on (i.e. a ratio $\mathrm{b} / \mathrm{a}=0.2$ ) and we do no further analyse also MaNGA 1-38802 because it is at a redshift considerably higher (i.e. $\mathrm{z}=0.11$ ) than the other $[\mathrm{O}$ III] undet galaxies in the sample. The remaining 8 [O III] undet galaxies are located at redshift between 0.04 and 0.06 and have masses between $10^{9.6}$ and $10^{10.8} \mathrm{M}_{\odot}$. We decide to include as a control sample 12 SDSS star-forming galaxies with similar mass and $[\mathrm{N} I I] /\left[\mathrm{O}_{\mathrm{II}}\right]$ range, whose emission line ratios lie along the median SDSS sequence of star-forming galaxies within the $[\mathrm{O}$ III $] / \mathrm{H} \alpha$ vs $[\mathrm{N} \mathrm{II}] /[\mathrm{O}$ II $]$ diagram, . The diagnostic diagram for the original Q18 sample and for the MaNGA galaxies considered in this analysis is presented in Figure 1. Our aim is to search for galaxies with regions which are in the quenching phase, using the same diagnostic used in SDSS (see Q18), but applied to each resolved galaxy regions.

\subsection{From MaNGA to pure-emission cube}

Starting from the MaNGA datacubes processed by the data reduction pipeline (DRP, Law et al. 2016), the final emission lines maps are obtained applying the following spectralfitting procedure, similar to that proposed by Belfiore et al. (2016):

(i) Increasing the signal-to-noise of the continuum. To create a pure-emission datacube, it is necessary to accurately subtract the stellar continuum from the original datacube. At first, the noise is corrected for the effect of the spatially correlated noise between adjacent spaxels, as discussed in García-Benito et al. (2015). Then, in order to increase the signal-to-noise ratio $(\mathrm{S} / \mathrm{N})$ of the continuum and at the same time preserve the spatial resolution, spaxels which $\mathrm{S} / \mathrm{N}$ lower than 10 in the restframe $4740-4840 \AA$ range are binned together with a Voronoi tessellation approach ${ }^{1}$ (Cappellari \& Copin 2003). Spaxels with undetected continuum (i.e. S / N $<2$ ) are not included in the binning, and they are no further considered in our analysis. The size of the bins is not forced to be larger than the typical MaNGA point spread function (PSF, i.e. 2.5 arcsec at FWHM, see Table 1), therefore, it is possible that adjacent bins are statistically correlated.

(ii) Fitting the continuum. In the spatially binned spectra the emission-lines and the strong sky-lines (i.e. O I $\lambda 5577$,

1 The Voronoi tessellation routine can be found at http:// www-astro.physics.ox.ac.uk/ $\sim \mathrm{mxc} /$ software.
$\mathrm{NaD} \lambda 5890$, O I $\lambda 6300, \mathrm{O}$ I $\lambda 6364)$ are masked within a window of $1400 \mathrm{~km} \mathrm{~s}^{-1}$. Then, the spectral continuum has been fitted choosing among various simple MILES stellar population models(Vazdekis et al. 2012) using penalised pixel fitting ${ }^{2}$ (pPXF, Cappellari \& Emsellem 2004) without taking into account dust extinction and using a set of additive polynomials up to the 4 th order to correct the continuum shape.

(iii) The pure-emission datacube. The best-fit continuum of each spatial bin is subtracted from the single original spaxels composing the bins, and the resulting data cube is composed by spaxels of pure-emission spectra.

\subsection{Emission-lines maps}

In this section, we describe the routine that we apply to pure-emission datacube to obtain maps of individual

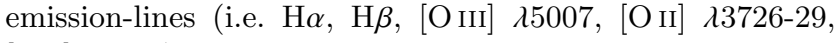
[N II] 26584).

(i) Increasing the signal-to-noise of nebular lines. $\mathrm{H} \alpha$ fluxes are measured in each spaxel from the pure-emission datacube. In order to reach an $\mathrm{S} / \mathrm{N}(\mathrm{H} \alpha)>5$ we perform a further Voronoi binning tessellation, not considering spaxels with $\mathrm{S} / \mathrm{N}(\mathrm{H} \alpha)<1$, which are no further considered in our analysis. This procedure allows studying nebular emission properties also in the outskirts of galaxies, at the cost of slightly worsening the spatial resolution. We find that no spaxels needs to be binned inside the effective radius of the analysed galaxies since their $\mathrm{S} / \mathrm{N}(\mathrm{H} \alpha)$ is always higher than 5. Therefore, the original central spatial resolution is preserved and dominated by the point spread function (PSF) of MaNGA datacubes, i.e. an area covered by almost 20 spaxels.

(ii) Fluxes and errors. In each spaxel, fluxes are measured by integrating the Gaussian best fit to the lines $\mathrm{H} \alpha, \mathrm{H} \beta$, [O III], [O II] (we consider [O II] = [O II $] \lambda 3726+[\mathrm{O}$ II $] \lambda 3729$ ), $[\mathrm{N} \mathrm{II}] \lambda 6584$ (hereafter $[\mathrm{N} \mathrm{II}]$ ), and [S II] $\lambda \lambda 6717-6731$. Errors on the fluxes are obtained by the propagation of errors on a Gaussian amplitude and standard deviation.

In our analysis, we need reliable measures of $[\mathrm{N}$ II] and $[\mathrm{OII}]$; hence the spaxels with $\mathrm{S} / \mathrm{N}<2$ in these lines are not considered either. Instead, since the fingerprint of the method is the weakness or lack of the [O III] emission, spaxels with $\mathrm{S} / \mathrm{N}([\mathrm{O} I I I])<2$ are kept as upper-limit values with $[\mathrm{O} I I I]=2 \times \sigma[\mathrm{O} I \mathrm{II}]$, where $\sigma[\mathrm{OIII}]$ is the error on the [O III] flux.

\subsection{The derived quantities from MaNGA data}

The maps of $\mathrm{H} \alpha, \mathrm{H} \beta$, [O III], [O II] and [N II], which form the starting point of our classification criteria (see subsection 2.4), are corrected for dust attenuation based on the $\mathrm{H} \alpha / \mathrm{H} \beta$ ratio. In order to perform a proper correction for dust extinction, spaxels with $\mathrm{S} / \mathrm{N}(\mathrm{H} \beta)<3$ and $\mathrm{S} / \mathrm{N}(\mathrm{H} \alpha)$ $<5$ are no further considered in the analysis. For the other spaxels, the colour excess $\mathrm{E}(\mathrm{B}-\mathrm{V})$ is derived adopting the Calzetti et al. (2000) attenuation law and assuming the Case $\mathrm{B}$ recombination and a Balmer decrement $\mathrm{H} \alpha / \mathrm{H} \beta=2.86$

2 pPXF code can be downloaded from http://www-astro. physics.ox.ac.uk/ ${ }^{\mathrm{mxc} / \text { software. }}$ 
(typical of $\mathrm{H}$ II regions with electron temperatures $\mathrm{T}_{\mathrm{e}}=10^{4}$ $\mathrm{K}$ and electron density $\mathrm{n}_{\mathrm{e}} \sim 10^{2}-10^{4} \mathrm{~cm}^{-3}$, Osterbrock 1989; Dopita \& Sutherland 2003) . Negative values of E(B-V) between about -0.05 and $\sim 0$ (i.e. inverted Balmer decrement, with $\sim 2.7 \leq \mathrm{H} \alpha / \mathrm{H} \beta<2.86$ ) are found in almost all galaxies in our sample, with percentages between $2 \%$ and $14 \%$ of the spaxels (but the galaxy 1-352114 shows $\mathrm{E}(\mathrm{B}-\mathrm{V})<0$ in $\sim 52 \%$ of its spaxels). However, these values are still compatible with case $\mathrm{B}$, though at electron temperatures between $10^{4}$ and $2 \times 10^{4} \mathrm{~K}$ (i.e. $2.74 \leq \mathrm{H} \alpha / \mathrm{H} \beta<2.86$, Hummer \& Storey 1987). We assign $\mathrm{E}(\mathrm{B}-\mathrm{V})=0$ to these spaxels.

The dust corrected fluxes are converted to luminosity surface densities $\left(\mathrm{erg} \mathrm{s}^{-1} \mathrm{kpc}^{-2}\right.$ ). Then, the SFR surface density $(\Sigma \mathrm{SFR})$ is derived using the dust corrected $\mathrm{H} \alpha$ luminosity surface density and adopting the Kennicutt (1998) conversion factor for Kroupa (2001) initial mass function (IMF):

$$
\Sigma \mathrm{SFR}=\Sigma\left(\mathrm{L}(\mathrm{H} \alpha) / 10^{41.28}\right)\left[\mathrm{M}_{\odot} \mathrm{yr}^{-1} \mathrm{kpc}^{-2}\right] .
$$

In order to obtain estimates of the ionisation parameter $\log \mathrm{U}$ and gas-phase metallicity $\mathrm{Z}$ from the observables, in the $[\mathrm{O}$ III $] / \mathrm{H} \alpha$ vs $[\mathrm{N} \mathrm{II}] /[\mathrm{O}$ II $]$ plane, we compared the observed values with a grid of theoretical values obtained with photo-ionisation models by $\mathrm{C} 17$. To do this, we interpolate the original models with a denser grid in which the theoretical Z spans from 0.004 to 0.04 with steps of 0.001 and $\log \mathrm{U}$ from -3.6 to -2.5 with steps of 0.01 . When a spaxel lies in a region of the diagram which is not covered by the models we assign the value linearly extrapolated (see Figure 6). This assumption has an impact on galactic regions with $\log ([\mathrm{NII}] /[\mathrm{OII}])$ higher than about -0.1 (e.g. spaxels in the central region of MaNGA 1-43012, see Figure 2). We stress that these estimates of metallicity are not obtained from a calibration of the $[\mathrm{N} \mathrm{II}] /[\mathrm{O} \mathrm{II}]$ or other emission line ratios (e.g. Nagao et al. 2006; Curti et al. 2017) but they are relative to the outcome of the photo-ionisation models by $\mathrm{C} 17$ and they are indicative for separating galaxies with different gas-phase metallicity and should be considered as relative values.

Redshifts, optical colors and effective radii $\left(\mathrm{R}_{50}\right.$, i.e. elliptical Petrosian 50\% light radius in SDSS r-band) are obtained from the NASA Sloan Atlas v1_0_1 (Blanton et al. 2011), while NUV band magnitude are taken from the Galaxy Evolution Explorer (GALEX, Martin et al. 2005). Stellar masses, total star-formation rates (SFR) are taken from the database of the Max Planck Institute for Astrophysics and the John Hopkins University (MPA-JHU measurements $^{3}$ ) as in Q18. We use also the SDSS morphological probability distribution of the galaxies provided by HuertasCompany et al. (2011), which is built by associating a probability to each galaxy to belong to one of four morphological classes (Scd, Sab, S0, E).

\subsection{The classification scheme}

In this Subsection, we present the classification scheme applied to the 20 MaNGA galaxies in our sample. We stress that none of the Q18 best candidates from SDSS are in the MaNGA catalogue. Thus, we do not expect to find galaxies

3 see http://wwwmpa.mpa-garching.mpg.de/SDSS/. in an advanced phase of quenching, but more likely galaxies which could have just started it.

In Figure 2 and Figure 3 we show the key information needed to characterise the sample, along with the gr-i images from SDSS. Starting from the maps of dust corrected $[\mathrm{O} \mathrm{III}] / \mathrm{H} \alpha$ (i.e. our observable for the ionisation status) and $[\mathrm{NII}] /[\mathrm{OII}]$ (i.e. the observable for the metallicity) of each galaxy, we build the spatially resolved $[\mathrm{O}$ III $] / \mathrm{H} \alpha$ vs $[\mathrm{N} \mathrm{II}] /[\mathrm{O} \mathrm{II}]$ diagnostic diagram for the quenching. We classify the spaxels into 4 groups according to their position on the plane compared to the SDSS distribution: (i) spaxels lying above the median curve of the SDSS, represent galaxy regions whose ionisation status is compatible with ongoing star formation; (ii) spaxels between the median and the $1 \sigma$ limit of the SDSS distribution, are regions characterised by slightly lower ionisation, though still compatible with emission due to star formation; (iii) spaxels between $1 \sigma$ and $3 \times 1 \sigma$ SDSS limits, are galactic regions in a grey area between star formation and quenching; (iv) spaxels lying below the $3 \times 1 \sigma$ limit of the SDSS distribution are galaxy regions which are likely experiencing the star formation quenching.

\subsection{The sample of Quenching Galaxy candidates and the sample of Star-Forming galaxies}

Once that all the spaxels within each galaxies have been classified, we define as QGs (i.e. Quenching Galaxy candidates), those galaxies which have at least $1.5 \%$ of their spaxels below the $3 \times 1 \sigma$ curve, representing a conservative excess of spaxels with respect to those expected below the $3 \times 1 \sigma$ (i.e. $\sim 0.13 \%$ ) for a star-forming galaxy. The QGs will be further analysed as galaxies with regions potentially undergoing the quenching.

In particular, we find 10 QGs galaxies which show such plausible quenching regions (see Figure 2). On the contrary, the other 10 galaxies do not show any sign of quenching, with the most of their spaxels lying above and along the median of the SDSS star-forming galaxies relation, as shown in Figure 3 . Hence, their behaviour in the $[\mathrm{O} \mathrm{III}] / \mathrm{H} \alpha$ vs $[\mathrm{N} \mathrm{II}] /[\mathrm{O}$ II $]$ diagram is consistent with that of a typical star-forming galaxy. We show in Figure 8 and in the on-line material that also their resolved BPT diagram confirms their star-forming nature. Hence, we can simply call them star-forming galaxies (SFs). In the following, we compare their properties (i.e. parameter of ionisation log U, gas-phase metallicity Z, star formation rate densities $\Sigma$ SFR, etc.) with those of the QGs ones.

The main global properties of the QGs and SFs are listed in Table 1. By construction, the two samples have a similar stellar mass and redshift range, with an average (and also median) mass of $10^{10} \mathrm{M}_{\odot}$ and a mean redshift of $\mathrm{z}$ $\sim 0.048$. However, we find that two SF galaxies (i.e. 1352114 and 1-197704) have a central $[\mathrm{N} \mathrm{II}] /[\mathrm{O} \mathrm{II}] \sim-0.6$, which is $\approx 2$ dex lower than the lowest QGs. Therefore, their gas-phase metallicity is considerably lower than the metallicity range of the QGs sample. We exclude these two objects, further analysing the remaining 8 SFs galaxies. In Figure 1 we report the position in the $[\mathrm{O} \mathrm{III]} / \mathrm{H} \alpha$ vs $[\mathrm{N} \mathrm{II}] /[\mathrm{O} \mathrm{II}]$ diagram of the SDSS measures of the galaxies in the two samples.

Both samples show, on average, a typical Sersic profile of disc galaxies (i.e. $<\mathrm{n}_{\text {Sersic }}>1.2-1.4$ ), and they show $<b / a>$ (i.e. the ratio between the semi-axis of the galactic 

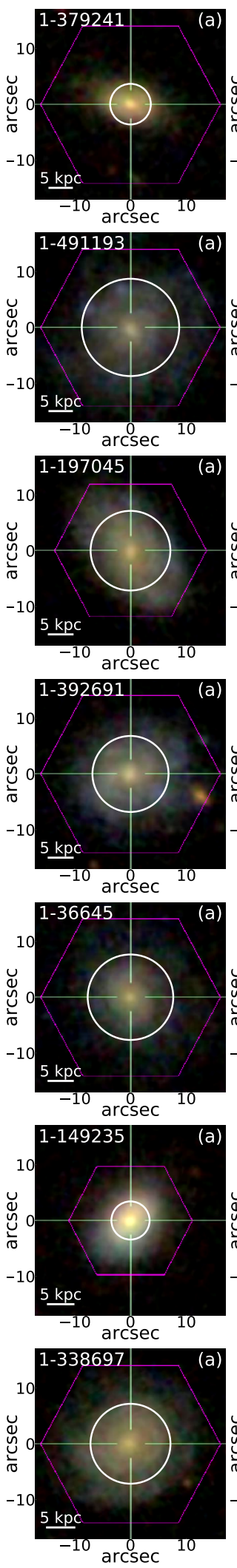
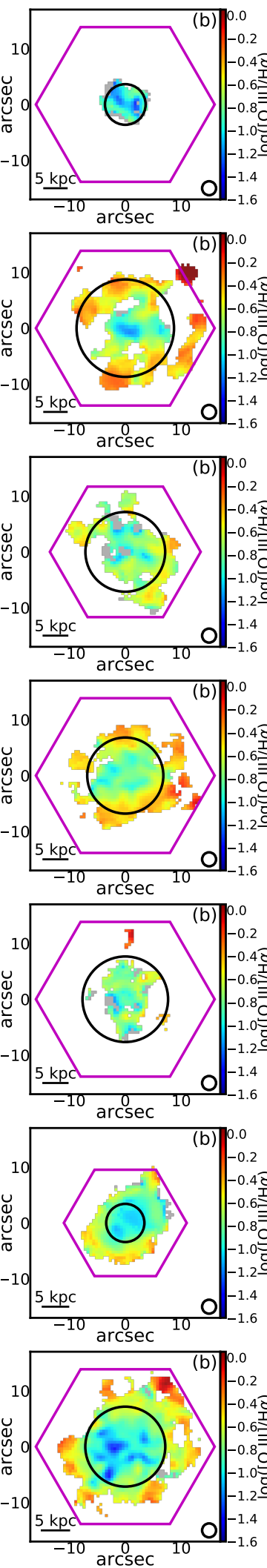
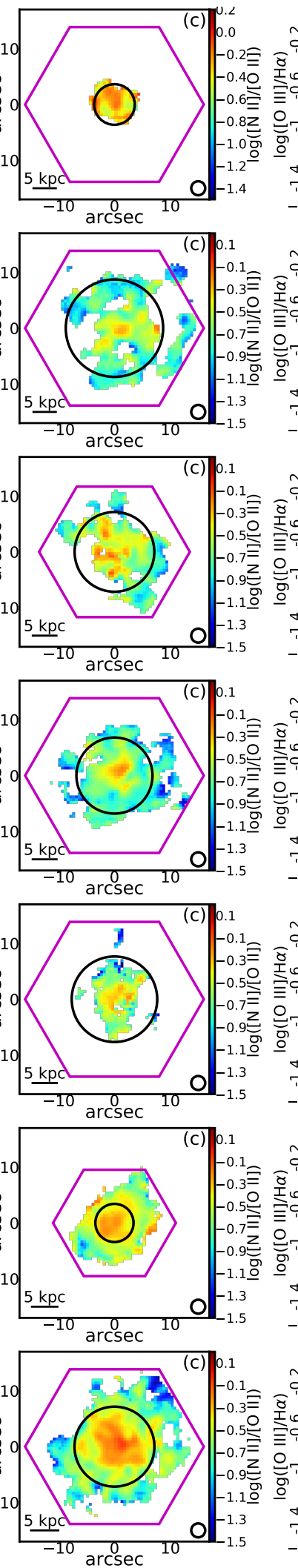
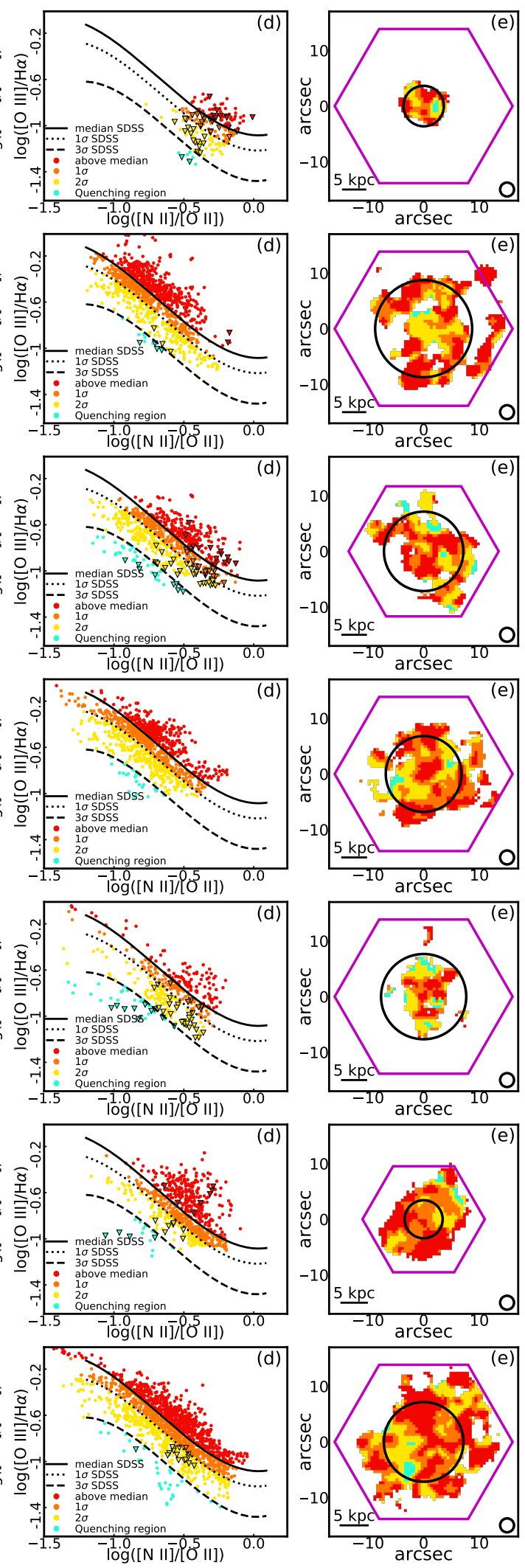

Figure 2. A summary of the 10 QG galaxies in our sample. (a) The g-r-i images composite from SDSS. Each image covers a region of $17 \times 17 \operatorname{arcsec}^{2}$ and in the bottom-left corner of each image is reported the scale of $5 \mathrm{kpc}$. (b) The dust-corrected [O III]/H $\alpha$ maps. The grey areas show regions with $\mathrm{S} / \mathrm{N}([\mathrm{O}$ III $])<2$. (c) The dust-corrected $[\mathrm{N} \mathrm{II}] /[\mathrm{O}$ II $]$ maps. (d) The $[\mathrm{O}$ III] $/ \mathrm{H} \alpha$ vs $[\mathrm{N}$ II $] /[\mathrm{O}$ II] diagnostic diagram for the quenching. The spaxels are colour-coded according to their position on the plane: red dots for those lying above the median curve, orange dots for them between the median and $1 \sigma$, yellow dots for spaxels which lie between $1 \sigma$ and $3 \times 1 \sigma$ and, finally, cyan dots for spaxels below the $3 \times 1 \sigma$ curve that, according with our classification criteria described in the text, represent likely quenching regions. The triangles represent spaxels with an upper limit in $\left[\mathrm{O}_{\mathrm{III}}\right] / \mathrm{H} \alpha$ (i.e. spaxels with $\mathrm{S} / \mathrm{N}([\mathrm{O}$ III $]$ ) < 2). (e) The map of the galaxies colour-coded according to the position of spaxels as in (d). In (a), (b), (c) and (e) the overlapped-magenta hexagonal shapes the MaNGA IFU bundles,

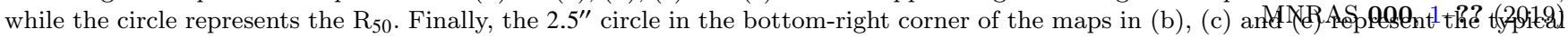
PSF (FWHM) of MaNGA data. 

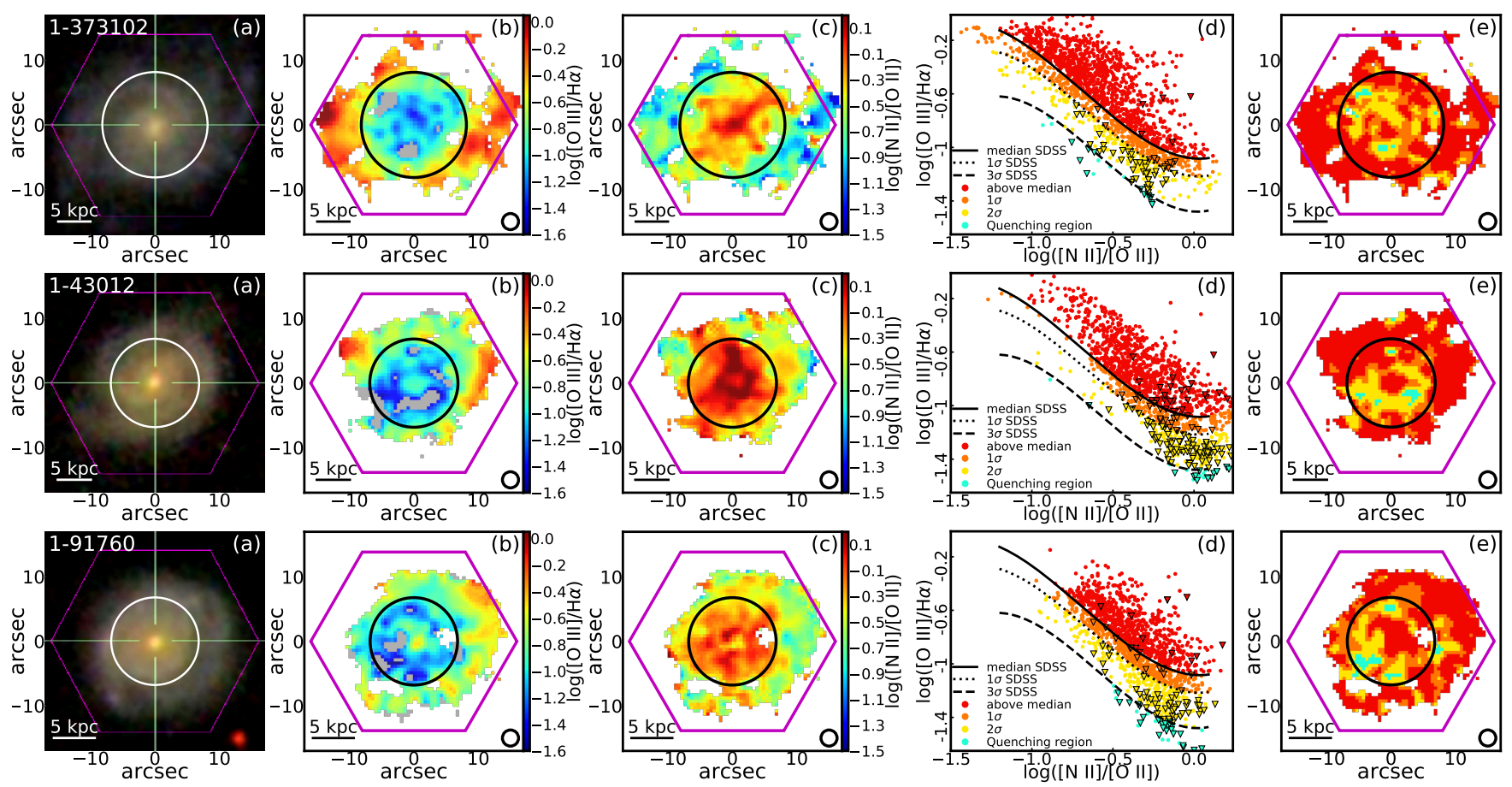

Figure 2. Continued.
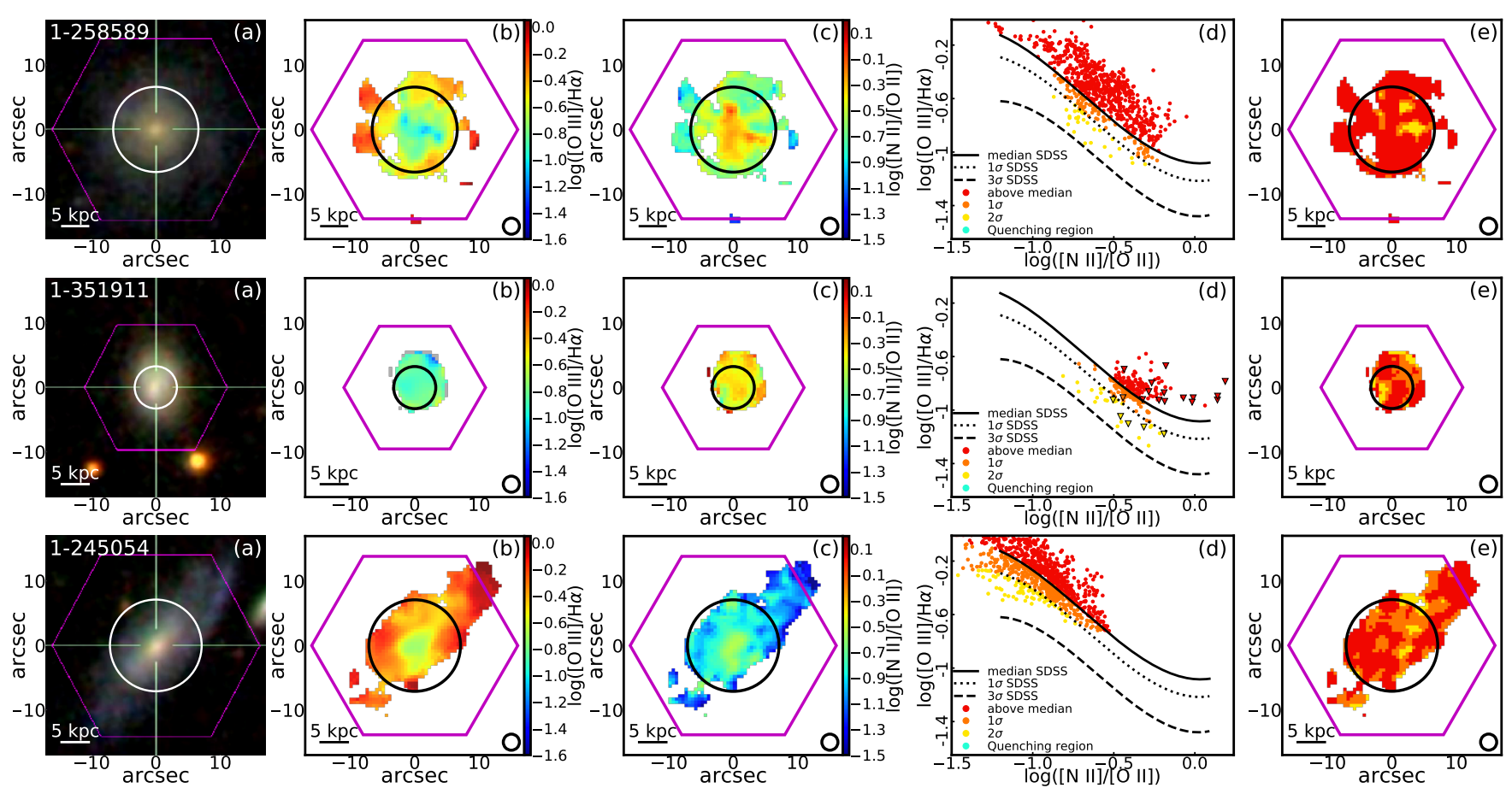

Figure 3. A summary of the $8 \mathrm{SF}$ galaxies in our sample. (a) The g-r-i images composite from SDSS. Each image covers a region of $17 \times 17 \operatorname{arcsec}^{2}$ and in the bottom-left corner of each image is reported the scale of $5 \mathrm{kpc}$. (b) The dust-corrected [O III]/H $\alpha$ maps. The grey areas show regions with $\mathrm{S} / \mathrm{N}([\mathrm{O} I \mathrm{II}])<2$. (c) The dust-corrected $[\mathrm{N} \mathrm{II}] /[\mathrm{O} \mathrm{II}]$ maps. (d) The $[\mathrm{O} \mathrm{III}] / \mathrm{H} \alpha$ vs $[\mathrm{N}$ II $] /[\mathrm{O} \mathrm{II}]$ diagnostic diagram for the quenching. The spaxels are colour-coded according to their position on the plane: red dots for those lying above the median curve, orange dots for them between the median and $1 \sigma$, yellow dots for spaxels which lie between $1 \sigma$ and $3 \times 1 \sigma$ and, finally, cyan dots for spaxels below the $3 \times 1 \sigma$ curve. The triangles represent spaxels with an upper limit in $[\mathrm{O}$ III] $/ \mathrm{H} \alpha$ (i.e. spaxels with $\mathrm{S} / \mathrm{N}([\mathrm{O}$ III $]$ ) $<2$ ). (e) The map of the galaxies colour-coded according to the position of spaxels as in (d). In (a), (b), (c) and (e) the overlapped-magenta hexagonal shapes the MaNGA IFU bundles, while the circle represents the $\mathrm{R}_{50}$. Finally, the $2.5^{\prime \prime}$ circle in the bottom-right corner of the maps in (b), (c) and (e) represent the typical PSF (FWHM) of MaNGA data. 

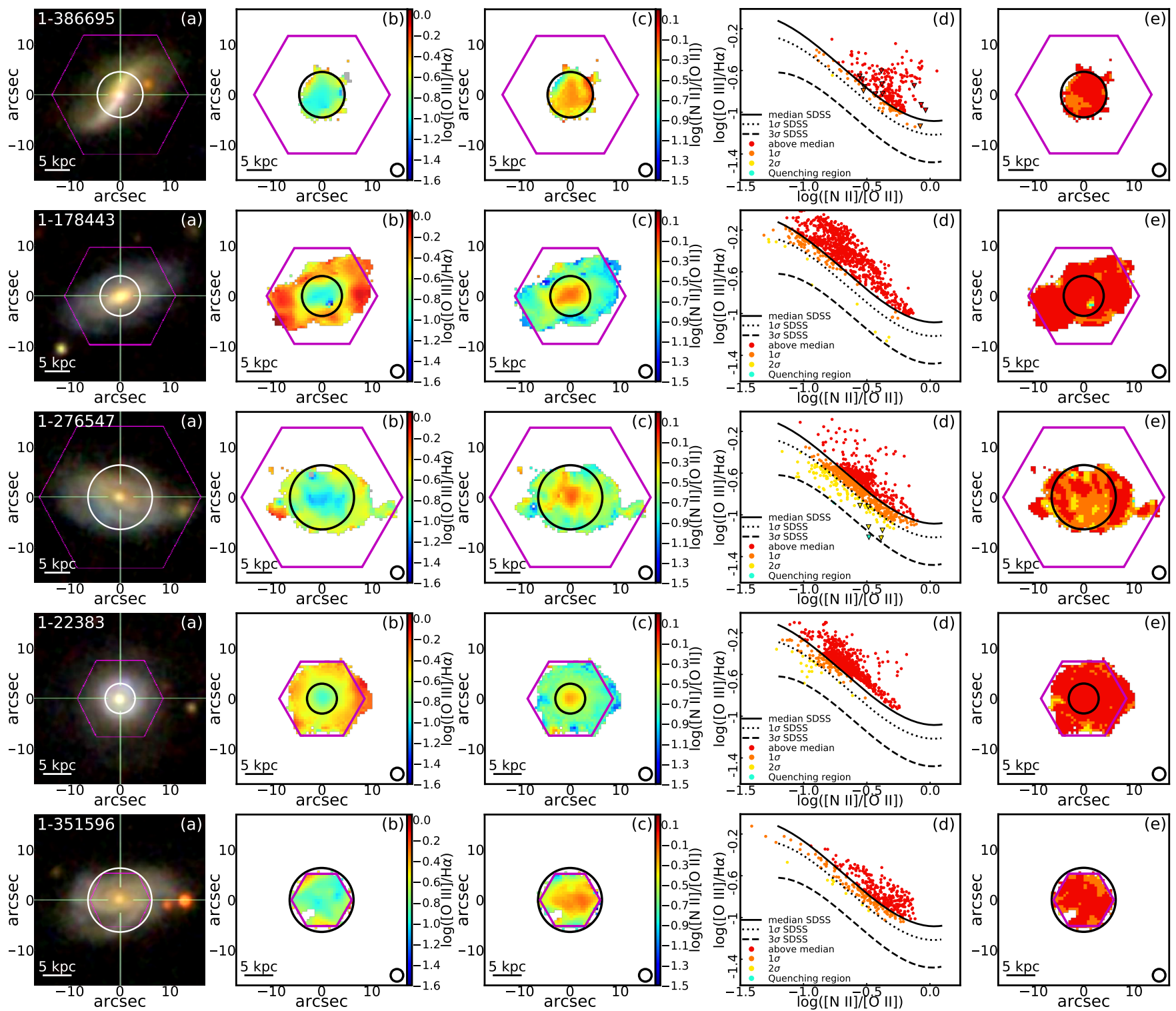

Figure 3. Continued.

plane) higher than $0.5-0.6$. Instead, we find differences in the specific-SFR (sSFR) and $R_{50}$ : the QGs have, on average, lower sSFR and larger $\mathrm{R}_{50}$ than the SFs ones. Moreover, we find that QGs have, on average, a slightly redder dust corrected colour $(\mathrm{u}-\mathrm{r})$ than SFs (i.e. $(\mathrm{u}-\mathrm{r}) \sim 1.4$ and $\sim 1.2$, respectively). Instead, QGs show a slightly bluer not dust corrected NUV-u colour than SFs (i.e. $\sim 0.7$ and $\sim 0.8$, respectively). It is not surprising that at these colours, galaxies of about $10^{10} \mathrm{M}_{\odot}$ lie below the Green Valley (e.g. Schawinski et al. 2014). In fact, the evolution of the colours in quenching galaxies is slower than that of the emission line ratios and it requires timescales larger than 1 Gyr to reach typical green valley colours (e.g. C17). Finally, it is interesting to note that stellar masses, colours, SFRs and the other parameters measured in QGs are consistent with those of the quenching candidates derived by Q18.

As mentioned earlier, we expect about $50 \%$ of the [O III] undet SDSS galaxies to be in quenching, and we find that 5 out of the 8 analysed [O III] undet galaxies belong to the QG sample, while the other ones are actually starforming galaxies. The discrepancy can be ascribed to an increased deepness of MaNGA data with respect to the SDSS ones, resulting in a still weak, but measurable [O III] (thanks to the higher $\mathrm{S} / \mathrm{N}$ ). Instead, it is interesting that 5 out of the 12 galaxies originally selected as star-forming are instead classified as QG galaxies. We will investigate the distribution of the quenching regions within QGs in the following sections. Here we mention that they are mainly placed offcentre, which explain why the regions inside the SDSS fibre have been classified as star-forming.

To summarise, according to the distribution of the spaxels on the $[\mathrm{O} \mathrm{III}] / \mathrm{H} \alpha$ vs $[\mathrm{N} \mathrm{II}] /\left[\mathrm{O}_{\mathrm{II}}\right]$ diagnostic diagram for the quenching, we obtain two MaNGA samples:

- QGs: 10 galaxies that show regions (at least 1.5\% of the total galaxy) satisfying our quenching criteria (i.e. lie below the $3 \times 1 \sigma$ of the SDSS star-forming distribution). 
Table 1. Main properties of our MaNGA QGs and SFs samples. In bold are indicated two galaxies analysed in detail in the text.

\begin{tabular}{|c|c|c|c|c|c|c|c|c|c|c|c|c|c|c|}
\hline sample & MaNGA-ID & $\mathrm{z}$ & RA & DEC & $\log \left(\mathrm{M}_{*} / \mathrm{M}_{\odot}\right)$ & $\mathrm{E}(\mathrm{B}-\mathrm{V})$ & $(\mathrm{NUV}-\mathrm{u})$ & (u-r) & $\begin{array}{c}\mathrm{Mg} \\
{[\mathrm{mag}]}\end{array}$ & $\begin{array}{l}\mathrm{sSFR} \\
{\left[\mathrm{yr}^{-1}\right]}\end{array}$ & $\begin{array}{c}\mathrm{R}_{50} \\
{[\operatorname{arcsec}]}\end{array}$ & $\begin{array}{l}\text { n (Sers.) } \\
\text { [arcsec] }\end{array}$ & $\mathrm{b} / \mathrm{a}$ & Morph. \\
\hline \multirow[t]{7}{*}{ QGs } & $1-379241$ & 0.0405 & 119.3 & 52.7 & 9.74 & 0.18 & 1.63 & 1.68 & -19.6 & -10.5 & 3.1 & 1.3 & 0.5 & Sab \\
\hline & $1-491193$ & 0.0405 & 171.5 & 22.1 & 9.61 & 0.20 & 0.16 & 1.26 & -19.3 & -10.4 & 8.6 & 1.3 & 0.9 & Scd \\
\hline & 1-392691 & 0.0435 & 156.2 & 36.0 & 9.75 & 0.00 & 0.55 & 1.44 & -19.8 & -9.9 & 6.4 & 1.3 & 0.8 & Scd \\
\hline & $1-36645$ & 0.0440 & 40.5 & -1.0 & 9.65 & 0.26 & 0.72 & 1.17 & -19.0 & -9.7 & 6.6 & 1.5 & 0.8 & / \\
\hline & $1-149235$ & 0.0464 & 169.3 & 51.0 & 10.21 & 0.29 & 1.02 & 1.31 & -20.1 & -10.1 & 3.1 & 1.3 & 0.7 & $\mathrm{Sab} / \mathrm{Scd}$ \\
\hline & $1-43012$ & 0.0527 & 112.9 & 38.3 & 10.48 & 0.33 & 0.76 & 1.56 & -20.5 & -10.5 & 6.2 & 1.1 & 0.8 & Scd \\
\hline & $1-91760$ & 0.0660 & 240.0 & 54.8 & 10.76 & 0.37 & / & 1.40 & -20.9 & -10.2 & 6.4 & 0.8 & 0.9 & Scd \\
\hline$<\mathrm{QGs}>$ & & 0.0478 & & & 10.05 & 0.25 & 0.67 & 1.37 & -19.9 & -10.2 & 6.1 & 1.2 & 0.8 & \\
\hline \multirow{3}{*}{ SFs } & $1-258589$ & 0.0405 & 186.7 & 44.9 & 9.72 & 0.35 & 0.56 & 1.14 & -19.4 & -10.0 & 6.7 & 1.6 & 0.9 & / \\
\hline & $1-351911$ & 0.0420 & 122.0 & 51.8 & 9.72 & 0.31 & / & 1.12 & -19.2 & -9.8 & 2.8 & 1.1 & 0.7 & Scd \\
\hline & $1-351596$ & 0.0554 & 118.6 & 49.8 & 10.41 & 0.36 & 0.99 & 1.33 & -21.0 & -10.1 & 5.3 & 0.9 & 0.5 & $\mathrm{Sab} / \mathrm{Scd}$ \\
\hline$<\mathrm{SFs}>$ & & 0.0473 & & & 10.08 & 0.30 & 0.76 & 1.19 & -20.2 & -9.9 & 4.4 & 1.5 & 0.6 & \\
\hline
\end{tabular}

- SFs: 8 star-forming galaxies which have same redshifts, stellar masses and gas-phase metallicity range of the QGs.

In section 4, we will extensively analyse the global behaviours of the two samples and we will compare their properties. In the next section, we will focus on the study of two galaxies, one for each sample, with the purpose of providing the details of the analysis that we performed on each galaxies in our sample.

\subsection{The impact of dust extinction on ionisation and metallicity indicators}

As shown in Q18, we can mitigate the U-Z degeneracy using the resolved $\left[\mathrm{O}_{\mathrm{III}}\right] / \mathrm{H} \alpha$ vs $[\mathrm{N}$ II $] /\left[\mathrm{O}_{\mathrm{II}}\right]$ diagram. The wavelength separation between the lines in the two ratios requires caution because of the not negligible effect of dust extinction. The classical approach relying on the Balmer decrement could be not accurate in recovering the intrinsic emission lines of an object deviating from the average star-forming galaxies. Other emission line ratios can be used which are less sensitive to this effect. For example, the $[\mathrm{O}$ III $] / \mathrm{H} \beta$ ratio would have the same sensitivity to the ionisation parameter of $[\mathrm{O} \mathrm{III}] / \mathrm{H} \alpha$ with the ad- vantages to be less affected by dust extinction. However, in order to guarantee a high level of precision in the ratio measurement, we should impose an $\mathrm{S} / \mathrm{N}(\mathrm{H} \beta) \geq 5$. This threshold would introduce a strong bias toward high SFR, penalizing the statistics of the quenching galaxies we are interested in selecting. Therefore, in order to evaluate the impact of dust extinction, we tested an alternative diagnostic diagram, with $[\mathrm{O}$ III $] / \mathrm{H} \beta$ not corrected for dust extinction (in place of dustcorrected $[\mathrm{O} \mathrm{III}] / \mathrm{H} \alpha$ ) vs dust-corrected $[\mathrm{N} \mathrm{II}] /[\mathrm{O} \mathrm{II}]$. Figure 4 shows the $[\mathrm{O}$ III $] / \mathrm{H} \beta$ vs $[\mathrm{N} \mathrm{II}] /[\mathrm{O}$ II] of QG $1-43012$. We find that the spaxels classified as quenching regions according to their position on the $[\mathrm{O} \mathrm{III}] / \mathrm{H} \alpha$ vs $[\mathrm{N} \mathrm{II}] /[\mathrm{O}$ II] diagram (i.e.

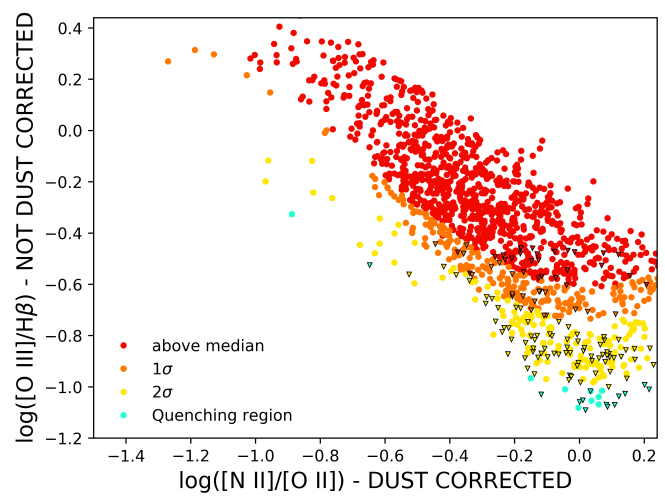

Figure 4. The resolved $\left[\mathrm{O} \mathrm{III}_{\mathrm{II}}\right] / \mathrm{H} \beta$ (not corrected for dust extinction) vs $[\mathrm{N} \mathrm{II}] /[\mathrm{O} \mathrm{II}]$ (corrected for dust extinction) diagram of QG 1-43012. The dots colour code is based on the position of each spaxel on the dust-corrected $[\mathrm{O}$ III $] / \mathrm{H} \alpha$ vs $[\mathrm{N} \mathrm{II}] /[\mathrm{O}$ II $]$ diagram, and it is the same as in Figure 1. The cyan is representing quenching regions, followed by the yellow for the galactic regions that lie between $3 \times 1 \sigma$ and $1 \sigma$ of the diagram, orange for those between $1 \sigma$ and the median and red for regions of pure starformation that are above the median of the diagram.

the spaxels lying below the $3 \times 1 \sigma$ of the SDSS relation, see Figure 2) remain those showing the lowest $[\mathrm{O}$ III $] / \mathrm{H} \beta$ values at fixed $[\mathrm{N} \mathrm{II}] /[\mathrm{O} \mathrm{II}]$. We find the same result also in the other QGs (see the online materials), hence we can state that our classification and results do not depend on the dust correction.

We note also that the $[\mathrm{O} \mathrm{III}] /[\mathrm{O} \mathrm{II}]$ ratio is sensitive to the ionisation status, as well. However, this ratio is also known to be rather sensitive to the gas-phase metallicity (e.g. Nagao et al. 2006), and it would be less effective to mitigate the U-Z degeneracy. Furthermore, the line ratio between [Ne III] $\lambda 3869$ and [O II] ([Ne III]/[O II]) is less af- 
fected by dust-extinction than [O III $] / \mathrm{H} \alpha$ and it is sensitive to the ionisation level of a star-forming galaxy. However, the [Ne III] line is usually faint to be detected at high $\mathrm{S} / \mathrm{N}$. Therefore, we are able to measure this line only in the central region of some galaxies in our SF sample. Finally, at larger wavelength, the line ratio [S III]/[S II] between the lines [S III] $\lambda \lambda 9060-9532$ and the doublet [S II] $\lambda \lambda 6726-6731$ is another ionisation tracer. The [S III] lines are measurable in MaNGA data up to redshift $\mathrm{z} \sim 0.08$, however, at the redshifts of our targets these lines end up in a spectral region dominated by a series of $\mathrm{OH}$ skylines, and therefore very difficult to be measured.

Similar remarks can be done about the metallicity indicator. We could, in principle, use different couples of emission lines closer in wavelength than $[\mathrm{N}$ II] and [O II], whose ratio is sensitive to the gas-phase metallicity. For example, $[\mathrm{N}$ II $] /[\mathrm{S} \mathrm{II}]$ shows a sensitivity to metallicity similar to the. However, the doublet [S II] $\lambda \lambda 6717-6731$ is considerably fainter than the [O II] $\lambda \lambda 3726-3729$ one and the cut in $\mathrm{S} / \mathrm{N}$ with $[\mathrm{N} \mathrm{II}] /[\mathrm{S} \mathrm{II}]$ would end up in excluding wider galactic area than with $[\mathrm{N} \mathrm{II}] /[\mathrm{O} \mathrm{II}]$.

Finally, we need to assess how much dust-obscuration corrections affect the measurement in the $[\mathrm{O}$ III $] / \mathrm{H} \alpha$ vs $[\mathrm{NII}] /[\mathrm{O} \mathrm{II}]$ diagnostic diagram. In Figure 1 and Figure 6 show the direction of dust vectors obtained by assuming the Calzetti et al. (2000) extinction law for an $\mathrm{E}(\mathrm{B}-\mathrm{V})=0.3$ (the direction is the same for the Cardelli et al. (1989) extinction law). The direction is almost parallel to the median, the $1 \sigma$ and $3 \sigma$ curves of the distribution and also to the iso- $U$ lines of the $\mathrm{C} 17$ models. This test guarantees that different dust laws do not affect our results.

Summarising, we can conclude that the $[\mathrm{O}$ III $] / \mathrm{H} \alpha$ vs $[\mathrm{N} \mathrm{II}] /[\mathrm{O} \mathrm{II}]$ diagram is robust against the Balmer decrement approach for correcting dust extinction and that these line ratios are the most suitable for mitigating the U-Z degeneracy.

\section{RESULTS I. A DETAILED CASE STUDY}

We will discuss the general results of the two populations in section 4, presenting individual details of the objects in our sample in the online materials. With the purpose of illustrating our research method, we show here the detailed analysis of two objects: QG 1-43012 representing an example of a QG, and SF 1-178443 among the galaxies in the SF sample. We choose SF 1-178443 because it has mass and redshift similar to those of QG 1-43012. This allows a direct comparison of the two systems, especially in terms of the ionisation parameter.

\subsection{Emission lines maps}

Figure 5 shows the r-band image, the $\mathrm{H} \alpha$ and [O III] luminosity surface density maps and the $[\mathrm{O}$ III $] / \mathrm{H} \alpha$ and $[\mathrm{N} \mathrm{II}] /[\mathrm{O}$ II] maps for the two galaxies. We find some differences, both structural and physical, between the two targets. They differ in size, being the SF smaller by a factor of $\sim 0.5$ than the $\mathrm{QG}$ one (i.e. $\mathrm{R}_{50} \sim 4.5 \mathrm{kpc}$ and $\sim 6 \mathrm{kpc}$, respectively) despite they have similar masses (i.e. $\log \left(\mathrm{M} / \mathrm{M}_{\odot}\right)=10.48$ and 10.35, respectively). The analysis of Figure 5 shows that:
- QG 1-43012 has some spiral arms in the r-band and. Therefore, according to the morphological probability distribution of the SDSS galaxies provided by Huertas-Company et al. (2011), it can be classified as a Scd galaxy. Instead, it remains difficult to see any significant spiral arm in the r-band image of SF 1-178443, while it shows a prominent bulge (or pseudo-bulge) and it has been classified as a Sab galaxy.

- The $\mathrm{H} \alpha$ emission is not homogeneously distributed in the QG, showing clumps which reach the maximum intensity of $\Sigma \log \mathrm{L}(\mathrm{H} \alpha) \sim 39.3 \mathrm{erg} \mathrm{s}^{-1} \mathrm{kpc}^{-2}$. The SF galaxy has a $\mathrm{H} \alpha$ distribution which is mostly concentrated and homogeneously distributed in the region inside the effective radius, where the emission reaches at values higher than $\Sigma \log \mathrm{L}(\mathrm{H} \alpha)$ $\sim 40 \mathrm{erg} \mathrm{s}^{-1} \mathrm{kpc}^{-2}$ and then degrades at lower values toward the outskirts.

- The QG has a globally weak emission in [O III], that rarely exceeds $\Sigma \log \mathrm{L}([\mathrm{O} \mathrm{III}]) \sim 38.5 \mathrm{erg} \mathrm{s}^{-1} \mathrm{kpc}^{-2}$ and, as a result, the $12.6 \%$ of its spaxels have an upper limit in [O III] (i.e. $\mathrm{S} / \mathrm{N}\left(\left[\mathrm{O}_{\mathrm{III}}\right]\right)<2$ ). Instead, the $[\mathrm{O} \mathrm{III}]$ emission of the SF galaxy follows the pattern of the $\mathrm{H} \alpha$ although being slightly weaker, as we expected since it arises from stellar ionising sources. In this case, only a few spaxels (i.e. $0.4 \%$ ) have $\mathrm{S} / \mathrm{N}([\mathrm{O}$ III $])<2$.

- The distribution of $[\mathrm{O} \mathrm{III}] / \mathrm{H} \alpha$ ratio (i.e. our ionisation level indicator) in the QG (see Figure 5) does not show a uniform gradient from the centre towards outer regions, but it reaches a minimum in an irregular annular region between $\sim 2$ and $\sim 5.5 \mathrm{kpc}$ (i.e. between $\sim 0.3$ and $\sim 0.9$ $\mathrm{R} / \mathrm{R}_{50}$ ) around the centre of the galaxy, then increasing towards more considerable distances. Instead, in the case of the $\mathrm{SF}$ galaxy, the $[\mathrm{O} \mathrm{III}] / \mathrm{H} \alpha$ shows a typical gradient with the $[\mathrm{O}$ III $] / \mathrm{H} \alpha$ raising from the centre towards the outskirts of the galaxy.

- The distribution of $[\mathrm{NII}] /[\mathrm{O} \mathrm{II}]$ (i.e. our metallicity indicator) shows an opposite behaviour with respect to $[\mathrm{O}$ III $] / \mathrm{H} \alpha$, in both QG and $\mathrm{SF}$ galaxies, with values increasing towards the inner parts of the galaxies. This relation between the $[\mathrm{O} \mathrm{III}] / \mathrm{H} \alpha$ and $[\mathrm{N} \mathrm{II}] /[\mathrm{O} \mathrm{II}]$ distribution is in part due to the well-known U-Z degeneracy between the ionisation parameter and gas metallicity (see Citro et al. 2017; Quai et al. 2018).

\subsection{The quenching diagnostic diagram}

\subsubsection{The $[\mathrm{O} \mathrm{iii}] / \mathrm{H} \alpha$ vs $[\mathrm{Nii}] /[\mathrm{O} \mathrm{ii}]$ diagram}

In Figure 6 we show the $[\mathrm{O}$ III $] / \mathrm{H} \alpha$ vs $\left[\mathrm{N}\right.$ II] $/\left[\mathrm{O}_{\mathrm{II}}\right]$ diagram of the two galaxies with the spaxels coloured according to their galactocentric distance and a grid of ionisation models by $\mathrm{C} 17$. The U-Z degeneracy is strongly mitigated, with the gas-phase metallicity $\mathrm{Z}$ increasing with $[\mathrm{N} \mathrm{II}] /[\mathrm{O}$ II] while the ionisation parameter $\log \mathrm{U}$ varying with $[\mathrm{O}$ III $] / \mathrm{H} \alpha$ at fixed metallicity. Results from Figure 6 suggest that a negative gradient of metallicity with radial distances is present in both galaxies. However, in SF 1-178443 at fixed metallicity, the ionisation parameter does not vary significantly while in QG 1-43012 it shows a large spread revealing differences in the ionising stellar populations in different regions of the galaxy. We stress that in this plane, at fixed values of $[\mathrm{N} \mathrm{II}] /[\mathrm{O} \mathrm{II}]$ (i.e. fixed metallicity), spaxels lying below the $3 \times 1 \sigma$ limit curve of the relation obtained from the star- 

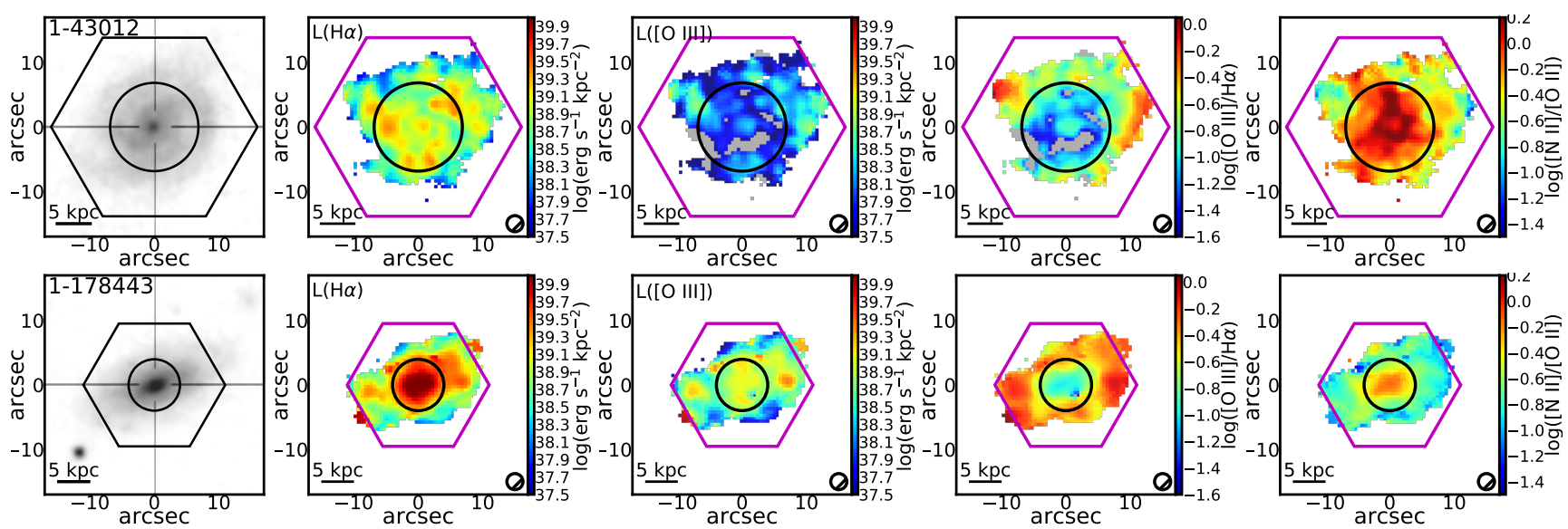

Figure 5. Spatially resolved maps of the two case galaxies. Each rows shows maps of QG 1-43012 (top) and maps of SF 1-178443 (bottom). Each column shows, from left to right (1) r-band images (2) luminosity surface density maps of dust-corrected H $\alpha$, (3) luminosity surface density maps of dust-corrected [O III], (4) dust corrected [O III]/H $\alpha$ maps and (5) dust corrected [N II]/[O II] maps. Spaxels coloured in grey represent regions with $\mathrm{S} / \mathrm{N}([\mathrm{O} \mathrm{III}])<2$ ). Overlapped in magenta are the hexagonal shapes of the MaNGA IFU bundles, while the black circles represent the $\mathrm{R}_{50}$. The $2.5^{\prime \prime}$ circle in the bottom-right corner of the maps represent the PSF (FWHM) of MaNGA datacubes. The galaxies go over the edge of the IFU shape because of the effect of the dithering, resulting in a coverage of a larger area of the sky.

forming population of SDSS represent regions compatible with the quenching. This region corresponds roughly to a $\log U<-3.4$. While in next sections we show in more details the $\mathrm{U}$ and $\mathrm{Z}$ profiles for our targets, from Figure 6 is already evident that the QG, on average, is more metallic than the SF one. About $72 \%$ of its spaxels have a supersolar metallicity (i.e. $\mathrm{Z}>0.02$ ), against $15.6 \%$ of the $\mathrm{SF}$ one. Moreover, the spaxels of the QG are spread across the entire plane covering the entire scale of ionisation levels, from $\log \mathrm{U}$ -2.4 to -3.6 . About $1.6 \%$ of its spaxels are in the quenching region below the $3 \times 1 \sigma$ curve of the SDSS distribution, and $14 \%$ of the spaxels lie between $1 \sigma$ and $3 \times 1 \sigma$. Instead, the $98 \%$ of the spaxels of the SF galaxy are in the pure starforming region, above the $1 \sigma$ curve of the SDSS distribution and with $\log \mathrm{U}$ higher than -3.2.

\subsubsection{The maps of the quenching regions}

In Figure 7 we show the contours of the resolved maps of the $[\mathrm{O} I I I] / \mathrm{H} \alpha$ vs $[\mathrm{N} \mathrm{II}] /[\mathrm{O} \mathrm{II}]$ diagram for the two galaxies. For QG 1-43012 the quenching regions cover an effective quenching area of $\sim 7.1 \mathrm{kpc}^{2}$, that becomes $\sim 67 \mathrm{kpc}^{2}$ wide if we include also the spaxels lying between $1 \sigma$ and $3 \times 1 \sigma$ as regions in which the quenching could be started. Being contiguous to the proper quenching regions, it is likely that the quenching has started also in these regions. This extended quenching region is mainly located in an irregular annulus around the centre of the galaxy.

Figure 8 shows the resolved diagnostic diagram of Baldwin et al. (1981, herafter BPT) for QG 1-43012 in which appears that the quenching regions are compatible with emission due to stellar ionisation, therefore, we can safely exclude the presence of an AGN. It should be noted that some spaxels, mostly located at the edge of the galaxy, lie above (but close) the BPT curve of Kauffmann et al. (2003) which distinguishes between galaxies where ionisation is due to star formation and the ones where it is due to AGN/LINER activity. These spaxels are observed in almost all the analysed galaxies (see the on-line material) and their behaviour is due to the uncertainties in $[\mathrm{O}$ III] $/ \mathrm{H} \alpha$. The emission lines, indeed, become weaker towards the outskirts of galaxies, increasing the uncertainties of the emission line ratio measurements. For example, the typical $\mathrm{S} / \mathrm{N}([\mathrm{O} \mathrm{III}] / \mathrm{H} \alpha)$ within $\mathrm{R}_{50}$ of $\mathrm{QG}$ $1-43012$ is between 4 and 25 , while it drops below 1.5 above $\sim 1.6 \mathrm{R}_{50}$.

\subsection{Radial profiles}

In this section, we extend the analysis of the two galaxies by investigating the radial profiles of the main quantities used in this work. We normalise the distance to the elliptical $R_{50}$, that we consider as a circular radius. Figure 9 shows the radial profiles of the colour excess $\mathrm{E}(\mathrm{B}-\mathrm{V})$ and the observables $[\mathrm{O} I I I] / \mathrm{H} \alpha$ and $[\mathrm{N} \mathrm{II}] /[\mathrm{O} \mathrm{II}]$, the radial profiles of the parameters $\log \mathrm{U}$ and gas-phase $\mathrm{Z}$ and that of the star formation rate density. Our findings can be briefly summarised as follows:

- The $\mathrm{E}(\mathrm{B}-\mathrm{V})$ radial profile of QG 1-43012 is quite scattered between $0 \leq \mathrm{E}(\mathrm{B}-\mathrm{V})<0.4$ at any radius. The spaxels marked as quenching regions show intermediate values of colour excess. The profile of SF 1-178443 is less scattered and it shows an almost flat median.

- As mentioned in the previous sections, the QG 1$43012[\mathrm{O} \mathrm{III}] / \mathrm{H} \alpha$ profile (i.e. ionisation level profile), shows a central peak, then it decreases down to a minimum $\log ([\mathrm{O} \mathrm{III}] / \mathrm{H} \alpha) \sim-1.2 \pm 0.2$ between $\sim 0.3<\mathrm{R} / \mathrm{R}_{50}<\sim 0.75$, and steeply increases again towards larger radii. This minimum corresponds to the region in which almost all the spaxels compatible with quenching are concentrated (see also Figure 7). Instead, SF 1-178443 shows a more homogeneous behaviour with a positive gradient in the $[\mathrm{O} \mathrm{III}] / \mathrm{H} \alpha$ profile, which is steeper at small radii, while it grows slowly at larger radii. Moreover, the $[\mathrm{O} I I I] / \mathrm{H} \alpha$ values are higher than those of QG 1-43012 at any radius.

- The profile of [N II]/[O II] for QGs follows a complementary pattern, with the off-centred peak set near 0.25 effective radius. The relation is tight in the centre's proximity, with 

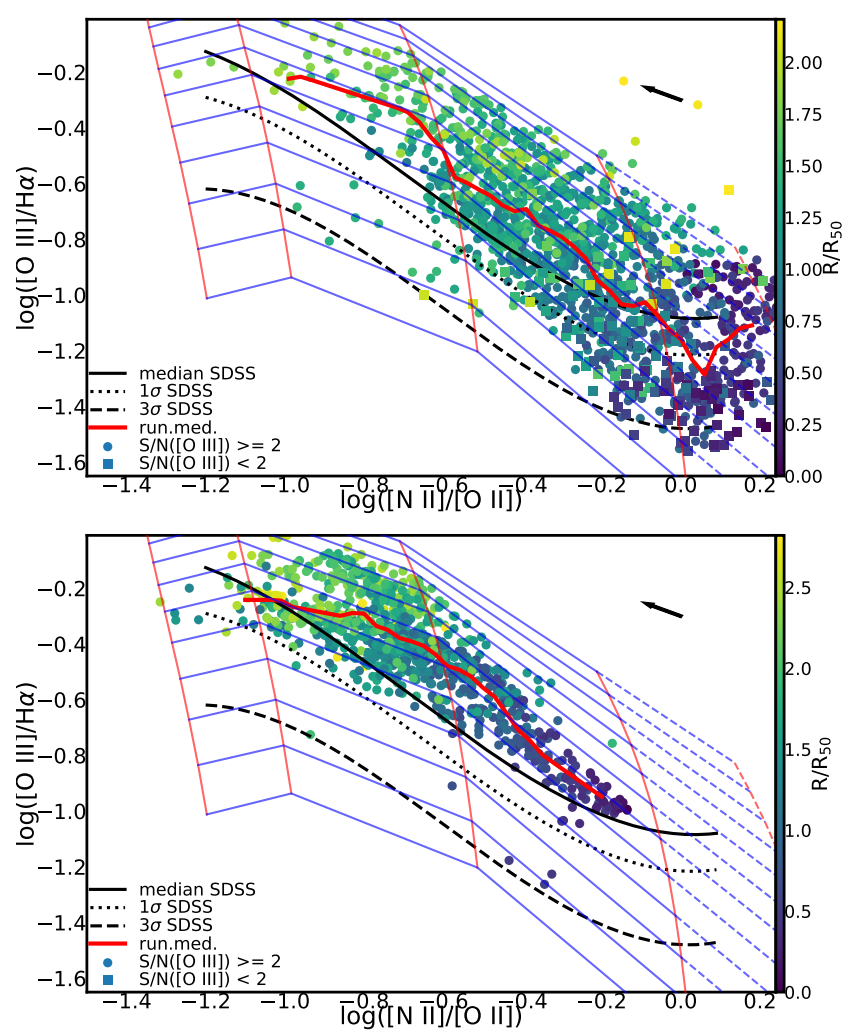

Figure 6. The resolved $[\mathrm{O} \mathrm{III}] / \mathrm{H} \alpha$ vs $[\mathrm{N} \mathrm{II}] /[\mathrm{OII}]$ diagram of $\mathrm{QG}$ 1-43012 (left) and SF 1-178443 (right). Each round dot represents a spaxel in which the $\mathrm{S} / \mathrm{N}([\mathrm{O} \mathrm{III}]) \geq 2$, while the square dots represent spaxels in which the $\mathrm{S} / \mathrm{N}\left(\left[\mathrm{O}_{\mathrm{III}}\right]\right)<2$ and their $[\mathrm{O} I \mathrm{II}] / \mathrm{H} \boldsymbol{\alpha}$ values are upper-limits. The colours of the dots change according to the distance $R / R_{50}$ of the spaxels from the centre of the galaxy. The red curve represents the running median (continue) of the relation. Instead, the black curves (polynomial of degree 4) represent the median (continue), $1 \sigma$ (dotted) and $3 \times 1 \sigma$ (dashed) of the distribution of SDSS star-forming galaxies (see Q18). Superimposed is reported the grid of photo-ionisation models by $\mathrm{C} 17$, with the red straight lines representing different metallicities (i.e. $\mathrm{Z}=\{0.004,0.008,0.02,0.04\}$ from left to right) and the blue straight lines representing different levels of the ionisation parameter $\mathrm{U}$ (i.e. from $\log \mathrm{U}-2.3$ in the top to -3.6 in the bottom). The blue and red dashed lines represent the model values linearly extrapolated beyond the coverage of the model grid up to $\mathrm{Z}=0.054$. The black arrow in the top-right corner represents the direction of the dust vectors for the Calzetti et al. (2000) extinction law, for an $\mathrm{E}(\mathrm{B}-\mathrm{V})=0.3$.

a $1 \sigma$ scatter of about 0.1 dex, but becomes higher than 0.2 dex at radii larger than $R_{50}$. The $S F$ galaxy shows lower $[\mathrm{N} \mathrm{II}] /[\mathrm{O} \mathrm{II}]$ values, that suggests lower metallicity than QG 1-43012 at any radius, with a maximum value in the centre that decreases rapidly approximately at $R_{50}$, then becoming almost flat.

- The $\log$ U profile of QG 1-43012 confirms the trend of the observable $[\mathrm{O} \mathrm{III}] / \mathrm{H} \alpha$, though with a larger spread. It peaks at the centre of the galaxy, then decreasing down to a minimum $\log \mathrm{U}=-3.2 \pm 0.1$ between 0.5 and $0.75 \mathrm{R}_{50}$ in correspondence of the $[\mathrm{O} \mathrm{III}] / \mathrm{H} \alpha$ minimum. At larger radii the profile increases again, though the spaxels are scattered through all the available ionisation levels between $\log \mathrm{U}-2.5$ and -3.5 . This findings suggests that the minimum in the ob-
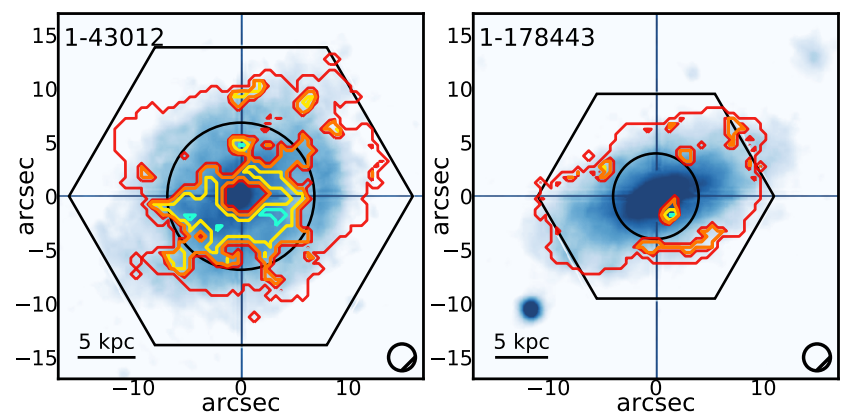

Figure 7. The contours of the resolved [O III]/H $\alpha$ vs $[\mathrm{N} \mathrm{II}] /[\mathrm{O}$ II $]$ diagram are superimposed to the $\mathrm{G}$ images (in false colours) of QG 1-43012 (left) and SF 1-178443 (right). The contours colour code is based on the position of each spaxel on the diagram, and it is the same as in Figure 1. The cyan is representing quenching regions, followed by the yellow for the galactic regions that lie between $3 \times 1 \sigma$ and $1 \sigma$ of the diagram, orange for those between $1 \sigma$ and the median and red for regions of pure star-formation that are above the median of the diagram. In the bottom-left corner is reported the scale of $5 \mathrm{kpc}$. Overlapped are the hexagonal shapes of the MaNGA IFU bundles, while the circles represent the $R_{50}$. The 2.5" circle in the bottom-right corner of the maps represent the PSF (FWHM) of MaNGA datacubes.

servable $[\mathrm{O}$ III $] / \mathrm{H} \alpha$ radial profile is due to a minimum in the ionisation level and not to the effect of the metallicity. It is worth mentioning that other QGs show these same features, though less clear (see online material and subsection 4.4).

Instead, the SF 1-178443 log U radial profile is almost flat up to $1.8 R_{50}$, with $\log U \sim-3.1$, suggesting that this star-forming galaxy is homogeneous in ionisation level and the increase of $[\mathrm{O} \mathrm{III]} / \mathrm{H} \alpha$ is due the decreasing of $[\mathrm{N} \mathrm{II}] /[\mathrm{O} \mathrm{II}]$, i.e. metallicity. In general, the spaxels are less scattered than those of the QG and only a handful of them have log U lower than -3.2 .

- The gas-phase metallicity radial profile of QG 1-43012 can be adequately studied only at radii larger than $0.5 R_{50}$. In fact, at closer distances, the metallicity estimate is linearly extrapolated from the $\mathrm{C} 17$ models grid beyond its $\mathrm{Z}=0.04$ limit, and up to $\mathrm{Z}=0.054$. At such high metallicity, a secondary nucleosynthesis origin of the nitrogen could explain this behaviour. In these circumstances, indeed, the $[\mathrm{NII}] /[\mathrm{O} \mathrm{II}]$ ratio (i.e. a tracer of the $\mathrm{N} / \mathrm{O}$ ratio) overestimates the oxygen abundances (i.e. $\mathrm{O} / \mathrm{H}$ ratio), leading to higher values of gas-phase metallicity. The resulting gas-phase metallicity radial profile follows a trend with a peak near $0.3 R_{50}$, that is similar to that of the observable $[\mathrm{N} \mathrm{II}] /[\mathrm{O} \mathrm{II}]$, and it is a typical metallicity profile found in galaxies with similar stellar mass (e.g., Sánchez et al. 2014). The $\mathrm{Z}$ radial profile of SF 1-178443 shows a negative gradient up to $1.25 \mathrm{R}_{50}$; then it becomes almost flat. In this galaxy, the gas-phase metallicity is lower than that of the QG one at any radius, and it shows a smaller spread.

- The log $\Sigma$ SFR radial profiles of the two galaxies are different, with QG 1-43012 having $\Sigma$ SFR values lower than those of SF 1-178443 at any radius. Its radial profile shows a weak negative gradient. A relative minimum is also visible around $0.3 R_{50}$, although less evident than that of the $[\mathrm{O}$ III $] / \mathrm{H} \alpha$ and $\log \mathrm{U}$ profiles. Instead, the $\Sigma \mathrm{SFR}$ radial profile of SF 1-178443 shows a negative gradient with a steeper slope until $\sim 1.25 \mathrm{R}_{50}$, then the profile becomes almost flat. 

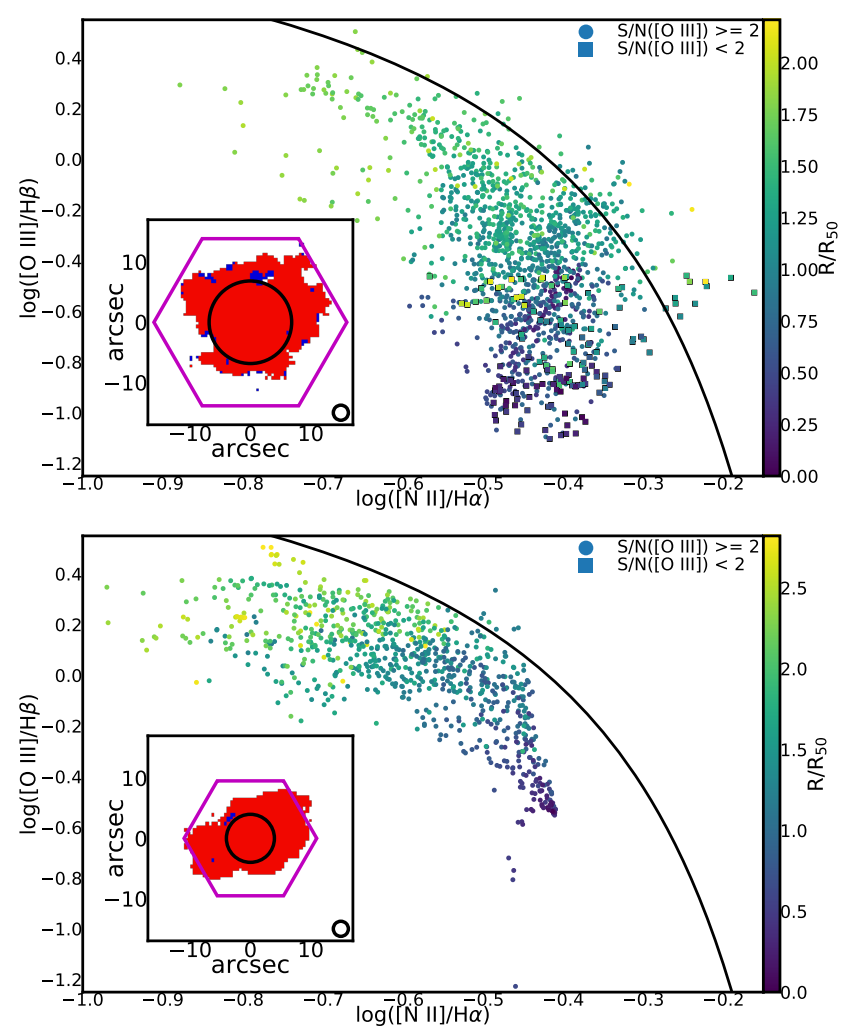

Figure 8. The resolved BPT diagram of QG 1-43012 (top) and of SF 1-178443 (bottom). Each round dot represents a spaxel in which the $\mathrm{S} / \mathrm{N}([\mathrm{O} I I]) \geq 2$, while the square dots represent spaxels in which the $\mathrm{S} / \mathrm{N}([\mathrm{O} I I I])<2$ and their $\left[\mathrm{O}_{\mathrm{III}}\right] / \mathrm{H} \boldsymbol{\alpha}$ values are upperlimits. The colours of the dots change according to the distance $R / R_{50}$ of the spaxels from the centre of the galaxy. The black curve is from Kauffmann et al. (2003). The coloured maps represent the resolved BPT maps of the two galaxies. Spaxels with ionisation dominated by star formation (below the Kauffmann et al. (2003) curve) are represented in red, while those whose the ionisation is dominated by AGN/LINERs radiation are represented in blue. Overlapped is the hexagonal shape of the MaNGA IFU bundle, while the circle represents the $\mathrm{R}_{50}$. The $2.5^{\prime \prime}$ circle in the bottom-right corner of the maps represent the PSF (FWHM) of MaNGA datacubes.

To summarise, the two galaxies show different characteristics, in terms of ionisation, gas-phase metallicity and distribution of star-formation rate surface density across the galactic plane.

\section{RESULTS II. GLOBAL COMPARISON OF QG AND SF GALAXIES}

In the previous section we gave details about the method we applied to each galaxy in our QG and SF samples. In this section, we present the general behaviour of the two samples. We stress that the two samples are in the same redshift range and they have same average stellar mass and central gasphase metallicity, therefore, we can directly compare their properties. We focus on the $\left[\mathrm{O}_{\mathrm{III}}\right] / \mathrm{H} \alpha$ vs $[\mathrm{N}$ II $] /[\mathrm{O}$ II $]$ diagram, and on the average radial profiles of the quantities we showed in the previous section (i.e. $\mathrm{E}(\mathrm{B}-\mathrm{V}),[\mathrm{O}$ III $] / \mathrm{H} \alpha$, $[\mathrm{NII}] /[\mathrm{O} \mathrm{II}], \log \mathrm{U}$, gas-phase Z, $\Sigma \mathrm{SFR})$. For each analysed property we define the significance as the distance of the average differences in units of $\sigma$, where $\sigma$ is the error in the average.

\subsection{The average $[\mathrm{O}$ iii $] / \mathrm{H} \alpha$ vs $[\mathrm{N}$ ii $] /[\mathrm{O}$ ii $]$ profile}

Figure 10 shows the average curves of $\mathrm{QG}$ and $\mathrm{SF}$ galaxies in the resolved $[\mathrm{O} \mathrm{III}] / \mathrm{H} \alpha$ vs $[\mathrm{N} \mathrm{II}] /[\mathrm{O} \mathrm{II}]$ diagram. The two samples share a very similar slope, compatible with the trend of the median distribution of the Q18 SDSS sample. However, they differ in normalisation, being the average curve of $\mathrm{SF}$ sample above the QG one at any $[\mathrm{N} \mathrm{II}] /[\mathrm{O}$ II $]$ value. The lower panel of Figure 10 shows the difference in $[\mathrm{O} \mathrm{III}] / \mathrm{H} \alpha$, as a function of $[\mathrm{NII}] /[\mathrm{OII}]$, between QGs and $\mathrm{SF}$ galaxies. We find an average difference of $-0.12 \pm 0.01$ dex with a significance over $10 \sigma$ level (see Table 2). The result does not change if we take the median in place of the average.

\subsection{The average $\mathrm{E}(\mathrm{B}-\mathrm{V})$ radial profile}

Figure 11 shows the average radial profiles of the colour excess $\mathrm{E}(\mathrm{B}-\mathrm{V})$ of the two samples. The SFs have higher extinction at any radius, with increasing values toward the centre. Figure 11 shows also the difference in $\mathrm{E}(\mathrm{B}-\mathrm{V})$ as a function of $\mathrm{R} / \mathrm{R}_{50}$, between $\mathrm{QG}$ and $\mathrm{SF}$ galaxies. We find that the average difference between QGs and SFs is about 0.05 and is confirmed at a significance of about $5 \sigma$ level (see Table 2). This result does not change by using the median in place of the average.

\subsection{The average radial profiles of $[\mathrm{O}$ iii $] / \mathrm{H} \alpha$ and $[\mathrm{N}$ ii $] /[\mathrm{O}$ ii $]$ ratios}

In Figure 12 we show the average radial profiles of [O III] $/ \mathrm{H} \alpha$ and $[\mathrm{N} \mathrm{II}] /[\mathrm{O} \mathrm{II}]$ ratios. At any radius, the $\mathrm{SF}$ sample shows, on average, higher $[\mathrm{O}$ III $] / \mathrm{H} \alpha$ and a slightly lower $[\mathrm{N}$ II $] /[\mathrm{O}$ II $]$ values than the QG population, though this one has larger errors. We find a difference in $[\mathrm{O} \mathrm{III}] / \mathrm{H} \alpha$ at a significance level of about $5 \sigma$ and a weak difference in $[\mathrm{N} \mathrm{II}] /[\mathrm{O}$ II] at a significance level slightly lower than $<3 \sigma$ (see Table 2 ). This result does not change if we use the median in place of the average.

\subsection{The average log U radial profile}

Figure $13 \log \mathrm{U}$ radial profiles for the two samples. The average $\log \mathrm{U}$ radial profile of $\mathrm{SF}$ galaxies increases very slowly from $\log \mathrm{U} \sim-3.2$ in the centre, toward $\sim-3.1$ in the outskirts. The QG galaxies log U profile, instead, has a maximum of $\log U \sim-3.1$ in the centre, then it decreases to a minimum $\log \mathrm{U} \sim-3.3$ around $0.5 \mathrm{R}_{50}$ before rising again to $\log \mathrm{U} \sim-3.1$ towards the outskirts. We note that 8 out to 10 QGs show such shape in their ionisation radial profile, while only $1 \mathrm{SF}$ galaxy shows a similar trend (see online material). On the contrary, only 1 QGs has a minimum in $\log \mathrm{U}$ in the center (QG 1-491193). Figure 13 shows the difference in $\log \mathrm{U}$ as a function of $\mathrm{R} / \mathrm{R}_{50}$, between $\mathrm{QGs}$ and $\mathrm{SF}$ galaxies. In the inner region there is no evidence of difference between the two samples, while they strongly differ between 0.3 and $1.2 \mathrm{R} / \mathrm{R}_{50}$. Therefore, the average 

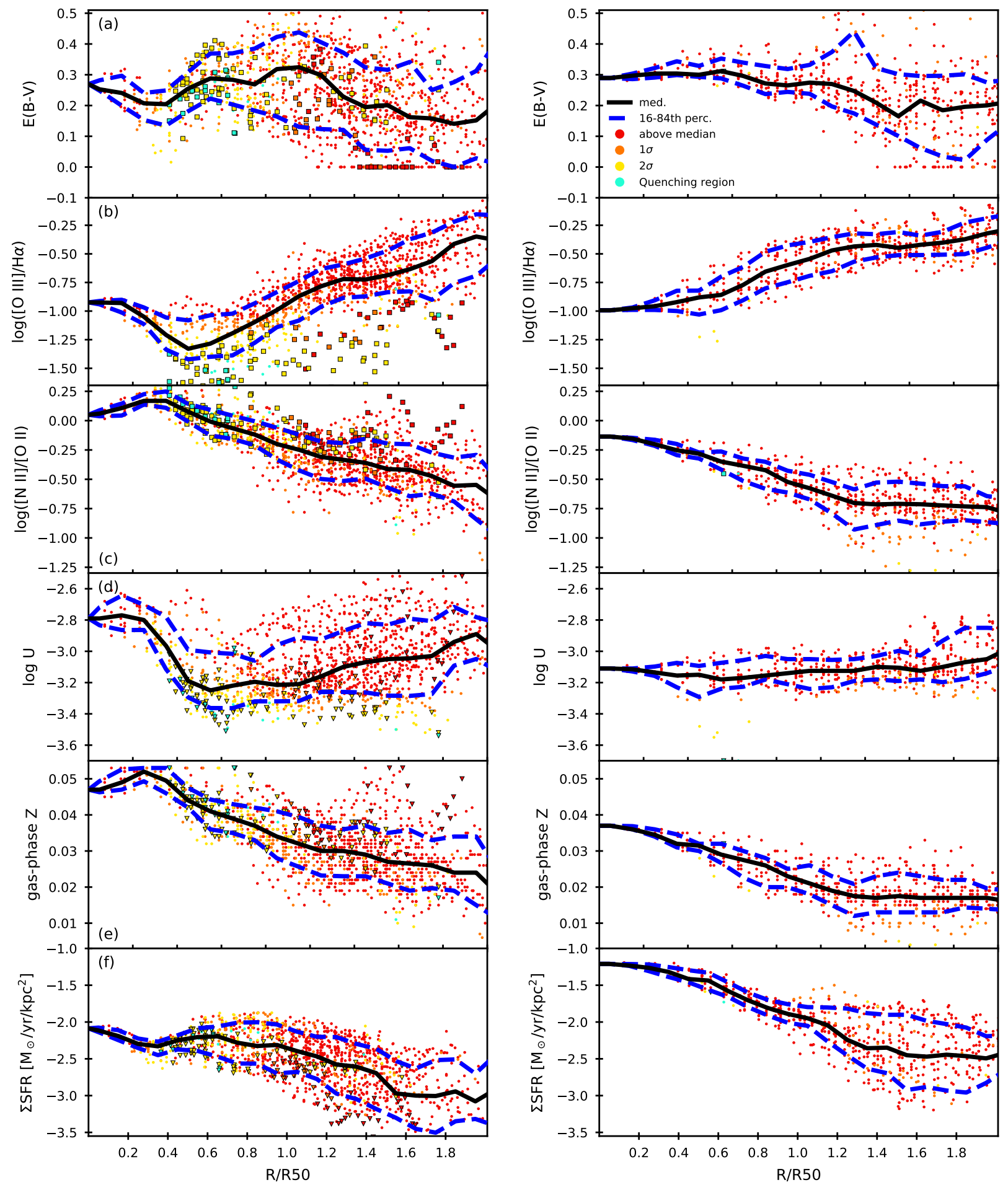

Figure 9. Radial profiles of QG 1-43012 (left) and of SF 1-178443 (right): (a) E(B-V), (b) dust-corrected [O III]/H $\alpha$, (c) dust-corrected $\left[\mathrm{N}_{\mathrm{II}}\right] /[\mathrm{O} \mathrm{II}]$, (d) $\log \mathrm{U}$, (e) gas-phase Z, (f) $\Sigma \mathrm{SFR}$. The black curves represent the median of the relations in bins of width $0.1 \mathrm{R} / \mathrm{R}_{50}$, while the blue ones represent the $16-84^{\text {th }}$ percentile of the relations. Each round dot represents a spaxel in which the $\mathrm{S} / \mathrm{N}([\mathrm{O}$ III $]) \geq 2$, while the square dots represent spaxels in which the $\mathrm{S} / \mathrm{N}\left(\left[\mathrm{O}_{\mathrm{III}}\right]\right)<2$ and their $[\mathrm{O} \mathrm{III}] / \mathrm{H} \alpha$ values are upper-limits. The dots colour code is the same as in Figure 7, and it is based on the position of each spaxel on the $[\mathrm{O} \mathrm{III}] / \mathrm{H} \alpha$ vs $[\mathrm{N} \mathrm{II}] /[\mathrm{O}$ II] diagram (Figure 1 and Figure 6). The cyan is representing quenching regions, followed by the yellow for the galactic regions that lie between $3 \times 1 \sigma$ and $1 \sigma$ of the diagram, orange for those between $1 \sigma$ and the median and red for regions of pure star-formation that are above the median of the diagram. 


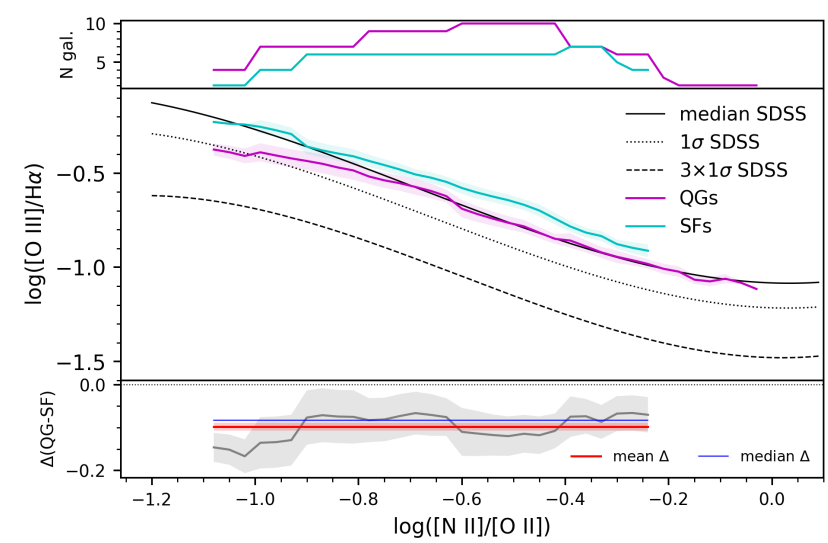

Figure 10. The average dust-corretced $[\mathrm{O}$ III $] / \mathrm{H} \alpha$ vs $[\mathrm{N} \mathrm{II}] /[\mathrm{O} \mathrm{II}]$ diagram for the QG and SF samples. Top: the number $\mathrm{N}$ of galaxies that contribute to the average. Centre: The magenta curve represents the mean curve of the QG galaxies, that we obtain by averaging their means; the magenta shaded area shows the error of the average (i.e. $\sigma_{\text {mean }} / \sqrt{N}$ ). The cyan curve shows the mean curve of the SF sample. The black curves represent median, $1 \sigma$ and $3 \times 1 \sigma$ limit of the SDSS star-forming galaxies sample (see Q18). Bottom: the grey curve represents the differences in $[\mathrm{O} I \mathrm{II}] / \mathrm{H} \boldsymbol{\alpha}$, as a function of $[\mathrm{NII}] /[\mathrm{OII}]$, between QGs and $\mathrm{SF}$ galaxies and the grey shaded area shows the propagated errors. The red line and pink shaded area represent the weighted mean and error of these differences averaged over the $[\mathrm{N} \mathrm{II}] /[\mathrm{O}$ II $]$ range (see Table 2).

difference is confirmed at a high significance of about $3.95 \sigma$ level (see Table 2).

\subsection{The average gas-phase metallicity radial profile}

The gas-phase metallicity radial profiles (see Figure 14) of the two samples have a similar slope and normalisation. We fit with a straight line to the average radial profile at galactocentric distances larger than $0.5 \mathrm{R}_{50}$ (as suggested in Belfiore et al. 2017b, to avoid smearing effects due to the MaNGA $\mathrm{PSF}$ in the inner part of galaxies) and smaller than $1.6 \mathrm{R}_{50}$. We obtain a slope of $\sim-0.01 / R_{50}$ for QGs (consistent with typical slopes of star-forming galaxies with similar stellar mass, e.g. Sánchez et al. 2014; Belfiore et al. 2017b), though the average profile of the QG galaxies shows a larger error than that of $\mathrm{SF}$ ones ${ }^{4}$. In this case, the difference between the sample is rejected (significance level $<3 \sigma$ ). This result confirms that the two samples have a similar gas phase metallicity.

\subsection{The average $\Sigma$ SFR radial profile}

From the analysis of the average radial profiles of the SFR surface density $(\log \Sigma \mathrm{SFR}$ ) of the two samples (see Figure 15) it turns out that, on average, in the central regions the SF galaxies show higher SFR density than QG, with a

${ }^{4}$ If we convert the gas-phase metallicity in terms of $12+\log (\mathrm{OH})$ (i.e. $12+\log (\mathrm{OH})=\log \left(\mathrm{Z} / \mathrm{Z}_{\odot}\right)+8.69$, with $\mathrm{Z}_{\odot}=0.02$ ), we will obtain a slope of $\sim-0.14 \mathrm{dex} / R_{50}$ for QGs.

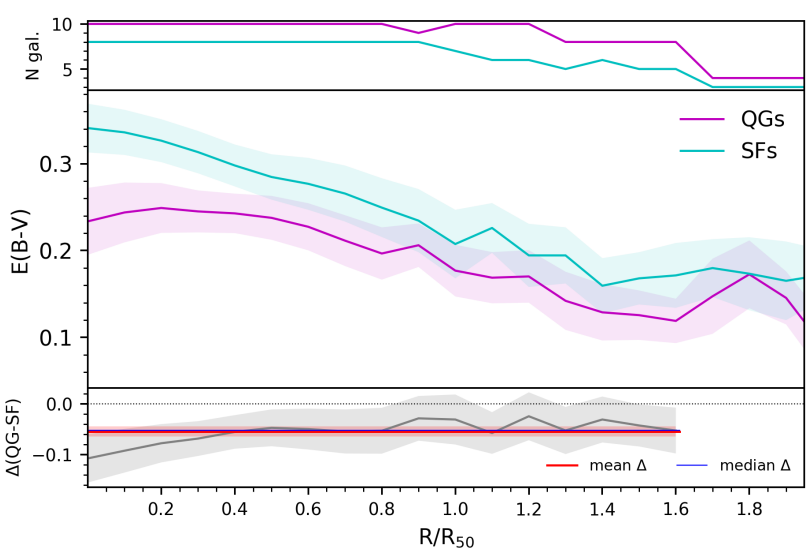

Figure 11. The average radial profiles of $\mathrm{E}(\mathrm{B}-\mathrm{V})$ for $\mathrm{QG}$ and $\mathrm{SF}$ samples. The colour code is the same as in Figure 10. The upper panels of the two plots show the number $\mathrm{N}$ of galaxies that contribute to the average. The grey curve in the lower panel of the two plots represents the differences in $\log \mathrm{U}$ and $\mathrm{Z}$, respectively, as a function of $R / R_{50}$, between $Q G s$ and $S F$ galaxies. The red line and pink shaded area represent the mean and error of these differences averaged over $\mathrm{R} / \mathrm{R}_{50}$ (see Table 2 ). We exclude from the significance analysis the trends at galactocentric distances larger than $1.6 \mathrm{R}_{50}$ due to the exiguous number of objects in our samples that extend at this radii.
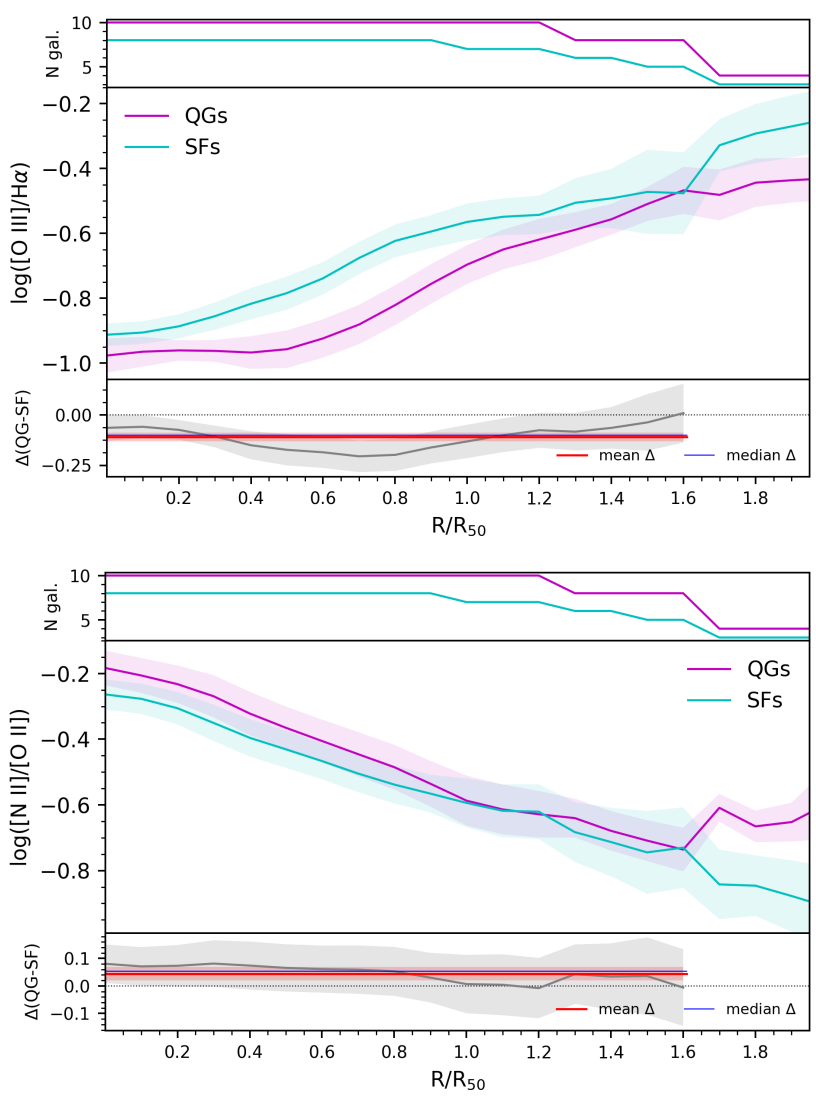

Figure 12. The average radial profiles of dust-corrected $\left[\mathrm{O}_{\mathrm{III}}\right] / \mathrm{H} \alpha\left(t_{o p}\right)$ and $[\mathrm{N} \mathrm{II}] /\left[\mathrm{O}_{\mathrm{II}}\right]$ (bottom) for QG and SF samples. The layout of the figure is the same as in Figure 10. 


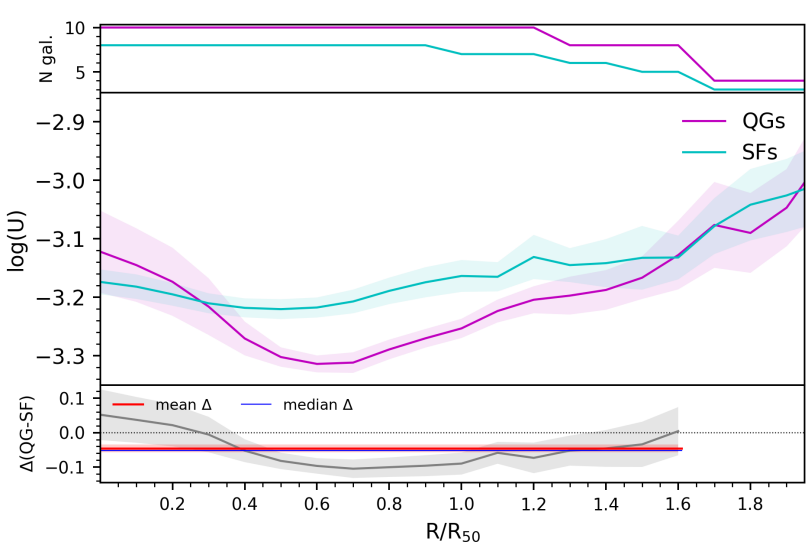

Figure 13. The average radial profiles of $\log \mathrm{U}$ for QG and SF samples. The layout of the figure is the same as in Figure 10.

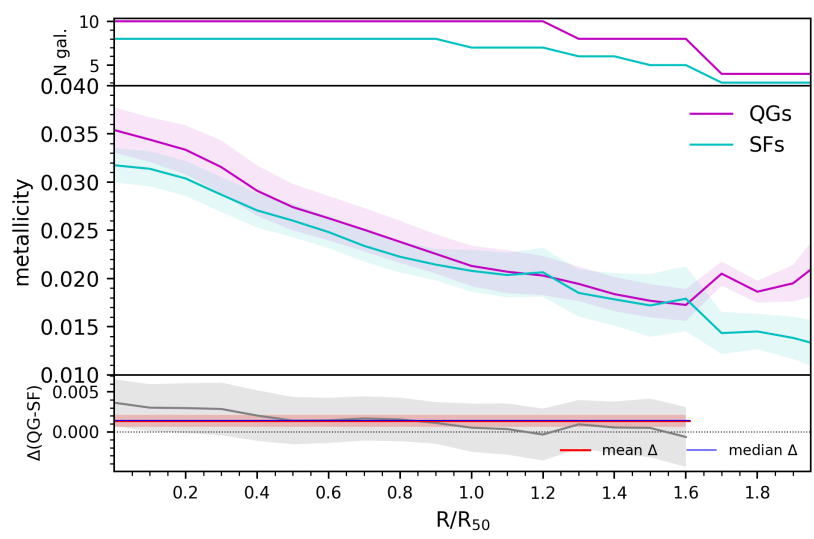

Figure 14. The average radial profiles of the gas-phase metallicity $\mathrm{Z}$ for QG and SF samples. The layout of the figure is the same as in Figure 10.

difference up to 0.6 dex. The average profile of QG galaxies shows a slow decline towards large radii. As shown in the central panel of Figure 15, the average SFR surface density of the SFs has an exponential profile, and its linear fit shows a slope of -0.67 (consistent with Spindler et al. 2018). Instead, the average trend of QGs shows suppression of SFR with respect to the exponential trend of SFs, with a linear fit characterised by a slope of -0.43 . The mean behaviour of the SFR in QGs is validated by the analysis of the trends of individual galaxies (see on-line material), with 9 out to 10 QGs showing an SFR suppressed at any radius with respect to the average trend of the SFs.

The lower panel of Figure 15 shows the difference in $\log \Sigma \mathrm{SFR}$ as a function of $\mathrm{R} / \mathrm{R}_{50}$, between QGs and $\mathrm{SF}$ galaxies. As expected, the difference is larger at small galactocentric distances and it becomes negligible in the outskirts. We find a strong differences in the median $\Sigma$ SFR between the two samples, of $\sim 0.4$ dex, with a significance at about $8 \sigma$ level (see Table 2 ).

It is interesting to discuss why the average star formation surface density radial profile of our QGs does not show a minimum similar to that of the ionisation parameter. The different behaviour of these two parameters can be due to the fact that the SFR, derived from the $\mathrm{H} \alpha$ luminosity, is

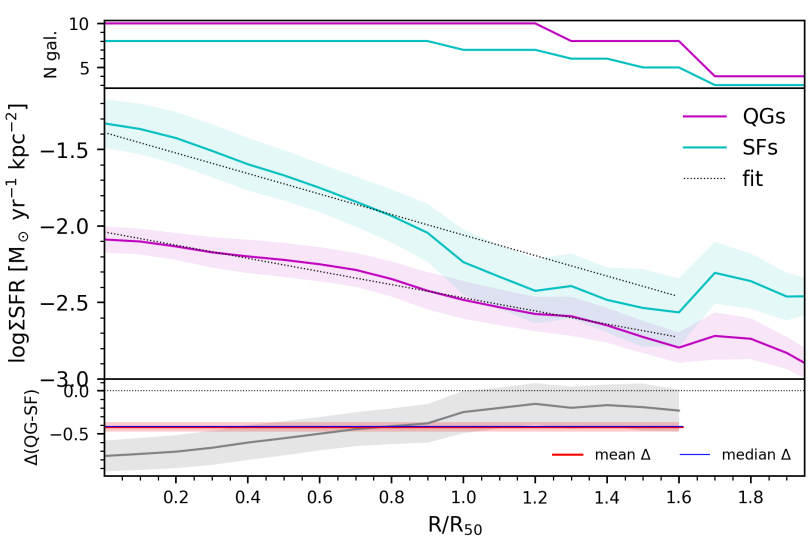

Figure 15. The SFR surface density ( $\log \Sigma \mathrm{SFR}$ ) radial profile for QG and SF samples. The dotted black lines represent the linear fit to the radial profiles. The layout of the figure is the same as in Figure 10.

Table 2. Median, mean, mean error and significance (expressed in units of $\sigma$ ) of the differences in the listed quantities between QGs and SF galaxies.

\begin{tabular}{lcccc} 
& median & mean & error & $\# \sigma$ \\
\hline$<\mathrm{E}(\mathrm{B}-\mathrm{V})>_{\mathrm{R}} / \mathrm{R}_{50}$ & -0.05 & -0.05 & 0.01 & 5.2 \\
$<\Delta \log ([\mathrm{O} \mathrm{III}] / \mathrm{H})>_{\left[\mathrm{N}_{\mathrm{II}}\right] /\left[\mathrm{O}_{\mathrm{II}}\right]}$ & -0.08 & -0.1 & 0.01 & 10.7 \\
$<\Delta \log ([\mathrm{O} \mathrm{III}] / \mathrm{H} \alpha)>_{\mathrm{R} / \mathrm{R}_{50}}$ & -0.1 & -0.1 & 0.02 & 5.2 \\
$<\Delta \log \left([\mathrm{N} \mathrm{II}] /\left[\mathrm{O}_{\mathrm{II}}\right]\right)>_{\mathrm{R} / \mathrm{R}_{50}}$ & 0.05 & 0.04 & 0.02 & 1.8 \\
$<\Delta \log (\mathrm{U})>_{\mathrm{R} / \mathrm{R}_{50}}$ & -0.05 & -0.05 & 0.01 & 3.95 \\
$<\Delta \mathrm{Z}>_{\mathrm{R} / \mathrm{R}_{50}}$ & 0.001 & 0.001 & 0.0007 & 1.8 \\
$<\Delta \log (\Sigma \mathrm{SFR})>_{\mathrm{R} / \mathrm{R}_{50}}$ & -0.4 & -0.4 & 0.05 & 7.9 \\
\hline
\end{tabular}

sensitive to the overall presence of $\mathrm{O}$ and $\mathrm{B}$ stars. Hence, its suppression represents an evidence of the general decrease of massive stars. Instead, as argued in the previous sections and in C17; Q18, the ionisation parameter promptly reacts to the disappearance of short-lived O stars, therefore, it is a better tracer of the actual distribution of quenching regions within galaxies. To confirm this, however, we need to discuss (see next section) the possibility that the absence of $\mathrm{O}$ stars can be connected with a stochasticity on the IMF in low SFR regime.

Several studies (e.g. Tacchella et al. 2015; Belfiore et al. 2018; Ellison et al. 2018; Morselli et al. 2018; Lin et al. 2019) interpret the suppression of the inner SFR in galaxies that lie below the star forming main sequence as an evidence of an inside-out quenching. But in that case, the quenching starts as the effect of a mechanism acting in the centre of the galaxies (e.g. AGN feedback) and then it propagates towards the outskirts. However, our method is not sensitive to AGN feedback (e.g. De Lucia et al. 2006; Fabian 2012; Cimatti et al. 2013; Cicone et al. 2014) as quenching mechanism, due to our a priori exclusion of AGNs. Also in our QGs we find a SFR density flatter than SF galaxies, suggesting a suppression of the SFR in the inner part of our galaxies. However, the ionisation parameter ( $\log \mathrm{U}$ ) has its minimum off-center, suggesting a quenching scenario more complex than an inside-out one. 


\subsubsection{Stochasticity on the initial mass function}

Lee et al. (2009) showed that assuming a Salpeter IMF, a conservative level of SFR of $1.4 \times 10^{-3} \mathrm{M}_{\odot} \mathrm{yr}^{-1}$ (i.e. $\log \mathrm{SFR}$ $=-2.8)$ is required to sustain the ability of robustly populate the entire IMF. Otherwise, the massive end of the IMF could result depleted by a certain amount, with the number of the most massive stars regulated by stochasticity. Therefore, for values of $\log \mathrm{SFR} \leq-3$ and fixed metallicity emerges a degeneracy between low [O III] flux due to incomplete sampling of the massive end of the IMF and the quenching of the star-formation. It is important to note that these kinds of studies regard the total SFR through the galaxy, especially dwarf ones. In our sample, the lowest total log SFR from SDSS data is $-0.79 \mathrm{M}_{\odot} \mathrm{yr}^{-1}$ (i.e. MaNGA 1-352114), however, with MaNGA we measure SFR on small scales (i.e. about a squared kpc per spaxel) and we can study the impact of stochasticity on the spaxels of our galaxies. In QG 1-43012 (i.e. the galaxy analysed in previews sections) the vast majority of the spaxels show $\log \Sigma \mathrm{SFR}<-2$ (see Figure 9) but only a small amount of $8 \%$ have $\log \Sigma \mathrm{SFR}<-3$ $\mathrm{M}_{\odot} \mathrm{yr}^{-1} \mathrm{kpc}^{-2}$. We observe a similar situation in the other QG galaxies (see Figure 15 and on-line materials).

Paalvast \& Brinchmann (2017) widely studied the impact of the stochastic sampling of the mass function on the production of lines requiring high energetic photons (i.e. [O III]) relative to that of the Balmer ones. All of their stochastic models predict a significant increase of the scatter of $[\mathrm{O} \mathrm{III}] / \mathrm{H} \beta$ ratios with decreasing of the $\mathrm{SFR}$ with respect to the typical BPT values, while at higher SFR the models well reproduce the BPT locus of the SDSS star-forming galaxies. For $\log \mathrm{SFR}<-3$, the lack of massive stars extends the scatter of $[\mathrm{O} \mathrm{III}] / \mathrm{H} \beta$ for solar metallicity from the BPT locus to values of $\log ([\mathrm{O} \mathrm{III}] / \mathrm{H} \beta)$ lower than -4 (i.e. $\sim-4.5$ using the $[\mathrm{O}$ III $] / \mathrm{H} \alpha$ ratio, alternatively), but also for $\log$ SFR -2 the scatter is considerably larger than that expected for a fully populated IMF. Moreover, the effect becomes more relevant with increasing metallicity.

In order to evaluate the impact of the stochasticity on our sample, we study galactic regions within our galaxies showing the lowest SFR values and super-solar gas phase metallicity. This combination of parameters maximises the effect in Paalvast \& Brinchmann (2017) models. To this aim, we gathered all the spaxels with $-3 \leq \log \Sigma$ SFR $<-2.5$ and super-solar metallicity (i.e. $0 \leq[\mathrm{N} \mathrm{II}] /[\mathrm{O} \mathrm{II}]<0.2$ ) and we analyse the distribution of their $[\mathrm{O}$ III $] / \mathrm{H} \alpha$. By definition, due to the stochasticity, we should obtain a wide distribution that covers the scatter due to the poor IMF sampling. We analyse an extreme conservative case in which all the spaxels showing an upper limit in [O III] (i.e. spaxels with $\mathrm{S} / \mathrm{N}([\mathrm{OIII}])<2$, about $22 \%$ of the spaxels in the bin) are considered with $\log [\mathrm{O} \mathrm{III}] / \mathrm{H} \alpha=-4$, as if all of them are the lowest outcome of the stochasticity models of Paalvast \& Brinchmann (2017). In Figure 16 we show the $[\mathrm{O}$ III] $/ \mathrm{H} \alpha$ distribution together with its cumulative curve. Even in this conservative situation, the median of the distribution is at $\log ([\mathrm{O} \mathrm{III}] / \mathrm{H} \alpha) \sim-1.1$, with a small scatter. Therefore, we can exclude the stochasticity on the IMF as the primary cause of low ionisation values observed in our galaxies. However, we cannot exclude that in a galaxy with recent quenching the low ionisation could be due both to stochasticity on the IMF sampling and the death of the most massive stars.
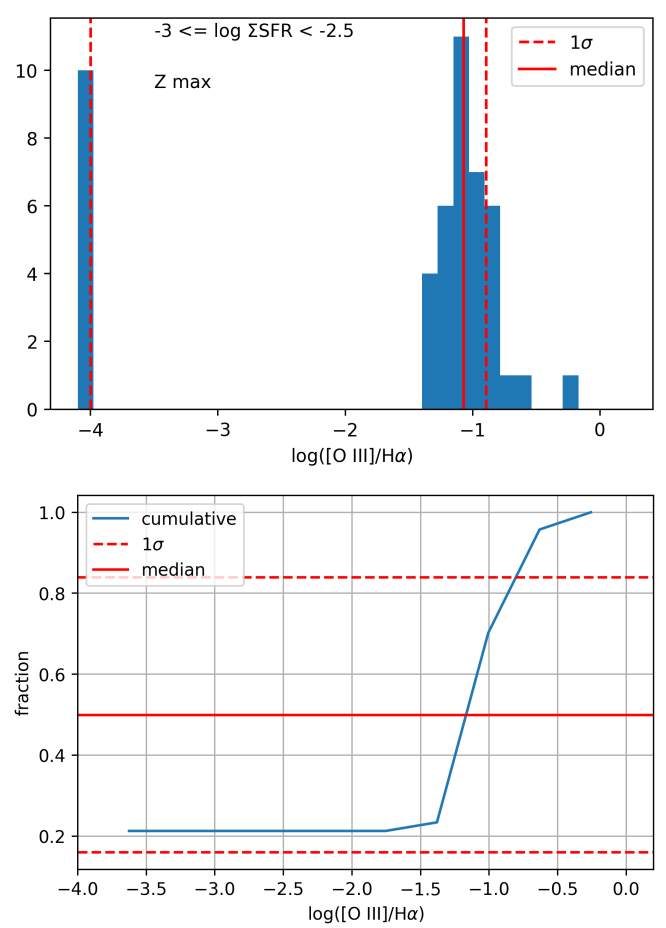

Figure 16. (Top:) the dust-corrected $[\mathrm{O}$ III $] / \mathrm{H} \alpha$ distribution for the spaxels with $-3 \leq \log \Sigma \mathrm{SFR}<-2.5$ and $0 \leq[\mathrm{N} \mathrm{II}] /[\mathrm{O} \mathrm{II}]<0.2$. The peak at $\left[\mathrm{O}_{\mathrm{III}}\right] / \mathrm{H} \alpha=-4$ represents the limit point that we choose for spaxels showing $\mathrm{S} / \mathrm{N}([\mathrm{O} \mathrm{III}])<2$. (Bottom:) the cumulative curve of the distribution.

\subsection{The spatial distribution of the quenching}

In this section, we focus on the QG galaxies with the aim to analyse the spatial distribution and extension of their quenching regions. None of the QGs shows extended regions compatible with our quenching criteria, instead they have groups of small regions ( $2-5$ regions each), with extension between 2 and $4 \mathrm{kpc}^{2}$, that are smaller than the MaNGA PSF (i.e. 2.5" of FWHM, or about $5 \mathrm{kpc}^{2}$ at these redshifts that corresponds to a percentage of the entire galaxies between $\sim 1 \%$ and $\sim 8 \%$ ). However, these groups of quenching regions are always interconnected in more extended areas that are characterized by slightly higher ionisation levels, and they lie in the region between $1 \sigma$ and $3 \times 1 \sigma$ of the $\mathrm{SDSS}$ data within the $[\mathrm{O} \mathrm{III}] / \mathrm{H} \alpha$ vs $[\mathrm{N} \mathrm{II}] /[\mathrm{O}$ II $]$ diagram (see Figure 1, and, for example the yellow area in Figure 7). Therefore, for effect of the PSF, it is likely that the actual size of the quenching regions is broader than that observed and it is mixed with the adjacent areas.

Figure 17 shows the total size of the likely quenching regions (i.e. the sum of the size of these regions) as a function of their average distance $\left(R / R_{50}\right)$ from the galactic centre of the QG galaxies. Only 2 out of 10 galaxies have significant quenching areas. They are QG 1-36645 and QG 1-197045 which have quenching regions covering an area of about $10 \%$ and $6 \%$ of the entire galaxies, respectively (i.e. corresponding to an area of about 10 and $9 \mathrm{kpc}^{2}$, respectively). The other 8 galaxies have quenching regions which cover between $\sim 1.1 \%$ and $\sim 6 \%$ in percentage of the entire galaxies (i.e. corresponding to an area between about 1.5 and $20 \mathrm{kpc}^{2}$ ). Moreover, all of them are located, on average, between 0.5 

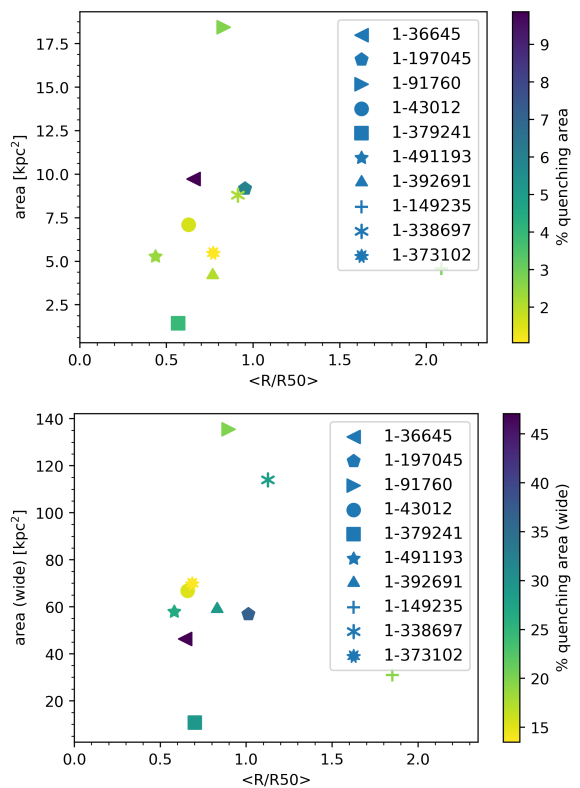

Figure 17. The total size of the likely quenching regions as a function of their average distance $\left(R / R_{50}\right)$ from the galactic centre of the QG galaxies. In the top panel we consider only the regions that satisfied our quenching criteria, while in the bottom panel we consider the area comprising the plausible quenching regions and the less extreme adjacent areas. The symbols represent different galaxies, while the colour represents the percentage of spaxels in the area with respect to the whole spaxels of the galaxy.

and $1 \mathrm{R}_{50}$ and only one of the galaxies have quenching regions in the outskirts at radii larger than $2 R_{50}$ (i.e. $Q G$ 1-149235).

It is interesting to consider, as an upper limit of the total size of the quenching distribution in these galaxies, the broader areas obtained by summing the dimension of quenching regions together with that of their adjacent less extreme areas. The result is shown in Figure 17. In this case, the quenching areas in QGs cover percentages between 13.5\% and $47 \%$ of all the spaxels, to which correspond sizes larger than $30 \mathrm{kpc}^{2}$ in 9 out of 10 QGs. Even when a broader area is considered, the average distance from the centre of the galaxies remains between about 0.5 and $1.1 \mathrm{R}_{50}$, confirming that only one of our galaxies have quenching regions in their center. Finally, we note that in 4 QGs these extended quenching regions shape an annulus, even if irregular and incomplete, around the galactic centre (see galaxies 1-392691, 1-373102, 1-43012 and 1-91750 in Figure 2).

How can a quenching mechanism be compatible with our findings of a off-centre start of the quenching? Several simulations (e.g. Prendergast 1983; Sellwood \& Wilkinson 1993; Regan \& Teuben 2003) show that in the inner regions of barred galaxies the gas flows toward the centre and it is often concentrated near the Lindblad resonance because of the dynamical interaction with the potential of the bar. There, the gas feeds intense episodes of star formation. However, we see neither bars nor rings of stars in the optical images of our galaxies. Nevertheless, even if the star formation is mostly in the ring, hardly ever it leads to form a ring of longlived stars (e.g. Kormendy \& Kennicutt 2004). Near-infrared images have shown that bars are hidden in approximately two-thirds of spiral galaxies, despite their appearance in optical wavelengths (e.g. Block \& Wainscoat 1991; Eskridge et al. 2002; Block et al. 2001; Laurikainen \& Salo 2002; Kormendy \& Kennicutt 2004). It is necessary to highlight that it is challenging to recognise bars and other galaxy substructures on the SDSS optical images of our QGs. However, since about 2/3 of star-forming galaxies show bars when observed at infrared wavelengths (see Kormendy \& Kennicutt 2004), it results therefore likely that a large fraction of our QGs should be composed by barred galaxies.

If there was a mechanism able to interrupt the inflow of gas toward the centre or the replenishment of gas from the surrounding hot halo, the star formation would continue by burning the remaining gas in the galaxy's reservoir. Tacconi et al. (2013) found that this reservoir can sustain the star formation for less than 1 Gyr before a typical star-forming galaxy runs out of gas. In these circumstances, at high star formation rates, the region of the ring should be the first to consume the fuel and to interrupt the star formation. Therefore, it is plausible that we are witnessing the very early phase of the quenching in our QGs. A possible interpretation of the results regarding the QGs, is that in these galaxies a quenching wave is presumably propagating from the inner region towards the outskirts because of the entire consumption of the available gas in intense episodes of star formation and we are witnessing the quenching phase on an annular region around the galactic centre.

It is interesting to report the recent results by Chen et al. (2019). In the MaNGA survey, they found a population of galaxies with ring-like post-starburst regions (RPSB). These regions are spatially distributed as the quenching regions in our QGs, and since the post-starburst phase traces timescales between $\sim 0.3-1 \mathrm{Gyr}$ after the quenching (i.e. stellar population dominated by A-type stars), the RPSB can represent a population of QG-like galaxies in a subsequent quenching phase. If we could confirm any affinity between RPSB and QG populations, we would prove that (i) there is a population of galaxies that experience a sharp and offcentre interruption of the star formation, and (ii) in these galaxies the quenching phase lasts $300-1 \mathrm{Gyr}$ (i.e. the poststarburst phase)

However, the exiguous number of galaxies in our sample, does not allow to establish whether our QGs really are progenitors of the RPSBs. Extending this study to the whole MaNGA population, also including a study of morphology, stellar and gas kinematic, would be critical to address this question.

\section{SUMMARY AND CONCLUSION}

In this paper, we present a spatially resolved study of 18 MaNGA galaxies (extracted from SDSS-IV MaNGA DR14, Bundy et al. 2015; Abolfathi et al. 2018), that is aimed at deriving spatial information about the quenching process within galaxies. For each galaxy, we obtain maps and radial profiles for SFR surface density (from the $\mathrm{H} \alpha$ luminosity corrected for dust extinction), $\mathrm{E}(\mathrm{B}-\mathrm{V})$ (from Balmer decrement), $[\mathrm{O} \mathrm{III}] / \mathrm{H} \alpha$ and $[\mathrm{N} \mathrm{II}] /[\mathrm{O} \mathrm{II}]$ emission lines ratios (corrected for dust extinction), and for ionisation parameters and gas-phase metallicity (derived from photoionisation models by $\mathrm{C} 17$ ). We classify the galaxies according to the 
spaxels distribution in the $[\mathrm{O}$ III $] / \mathrm{H} \alpha$ vs $[\mathrm{N} \mathrm{II}] /[\mathrm{O}$ II] diagram, extending the method devised by Q18 to IFU data, and we obtain two samples:

- QGs sample: 10 galaxies which show regions compatible with a recent quenching of the star formation. These quenching regions are those satisfying the criteria devised in Quai et al. (2018), showing [O III]/H $\alpha$ ratios that are too low to be explained by metallicity effects.

- SFs sample: a control sample of 10 galaxies which are compatible with ongoing star-formation.

The galaxies in the two samples are in the same mass and redshift ranges. However, in our analysis we exclude 2 lowmetallicity SFs to preserve also the same gas-phase metallicity range (see Table 1 for a list of the main properties of the two samples).

We discuss the general characteristics of QG and SF galaxies concerning gas-phase metallicity, ionisation status, color excess $\mathrm{E}(\mathrm{B}-\mathrm{V})$ and SFR. Our main results are summarised as follows:

- The average gas-phase metallicity radial profile of QG galaxies is slightly higher than that of SF ones at any radius. However, the difference between the two profiles is not significant. This result confirms that the two samples have a statistically similar gas-phase metallicity. This entails that the following results cannot be ascribed to metallicity effects.

- The average ionisation parameter log U radial profile of the SF sample shows a slow increase of log U towards the outskirts of the galaxies. Despite QGs show central log U values similar to those of $\mathrm{SFs}$, their $\log \mathrm{U}$ profile drops at radii $\sim 0.2 \mathrm{R} / \mathrm{R}_{50}$ and reaches a minimum at effective radii between 0.5 and 0.8 . This trend reveals a lack of $\mathrm{O}$ stars in the region surrounding the galactic center of the analysed QGs, suggesting that the quenching could be originated offcentre. We confirm the difference between the QGs and the SFs at a high significance level of about $5.5 \sigma$.

- The average radial profile of the star formation rate surface density of QGs is lower than that of the SFs, at any radii, suggesting an overall suppression of the star-formation rate. The difference is larger approaching small radii. As expected, this trend is similar to that of the colour excess $\mathrm{E}(\mathrm{B}-\mathrm{V})$. We confirm the difference between the QGs and the SFs at a high significance level of about $8 \sigma$.

- The quenching regions within our QGs are located between 0.5 and 1.1 effective radii from the centre and occupy a total area between $\sim 15 \%$ and $\sim 45 \%$ (i.e. between $\sim 10$ and $\sim 140 \mathrm{kpc}^{2}$ of the total galactic area ${ }^{5}$ ). It is interesting to note that none of these quenching regions is found in the inner part of our QGs, despite the low level of the measured SFRs. The recent findings by Chen et al. (2019) of a population of MaNGA galaxies showing post-starburst phase in a ring-like region spatially distributed as the quenching regions in our QGs, encourage us to analyse affinities between the two populations, and to study the QGs as progenitors of post-starburst galaxies.

We stress that we do not expect to find galaxies in an advanced quenching phase among the few analysed QGs,

5 When we refer to the total galactic area, we mean the total area of all the spaxels covering a galaxy. since they are not as extreme as the quenching candidates found by Q18, which should provide decisive clues on the early phase of the quenching. However, it can be expected that the quenching will propagate from the off-centre regions over all the galaxy. We interpret the off-centre distribution of quenching regions in QGs as an early phase of the quenching. In the case of no replenishment of new gas, the galactic regions with low gas depletion rapidly run out of fuel (e.g. Tacconi et al. 2013). Therefore, the quenching distribution in regions around the galactic centre of our QGs suggests that a shortage of cold gas started recently in the proximity of Lindblad in barred galaxies. There, the depletion time is shorter because of the accumulation of gas gathered by the effect of the gravitational potential of the bar, that induces high star formation rates (e.g. Kennicutt \& Evans 2012). Although it is challenging to recognise bars and other galaxy substructures in the SDSS optical images of our QGs, it turns out that about $2 / 3$ of star-forming galaxies show bars when observed at infrared wavelengths (Kormendy \& Kennicutt 2004). Therefore, it is likely that a large fraction of our QGs should be composed by barred galaxies.

A critical question that needs to be addressed is whether the QGs are actually starting the permanent quenching phase, or if the quenching regions they host are indicative of a minimum in the star formation history of the galaxy due to the interruption of inflows of fresh gas (see Wang et al. 2019 , for a discussion). In a future perspective, an extension of our method to the whole sample of galaxies in the SDSS IV MaNGA data release 15 (Aguado et al. 2019), together with a multi-wavelength sample including maps of the distribution of the cold gas would help to disentangle the two possibilities and shed lights on the mechanism driving the quenching.

\section{ACKNOWLEDGEMENTS}

The authors thank the anonymous referee for helpful suggestions and constructive comments. We are grateful to Filippo Mannucci and Roberto Maiolino for useful suggestions and discussions. The authors also acknowledge the grants PRIN MIUR 2015, ASI n.I/023/12/0 and ASI n.2018-23-HH.0. JB acknowledges support by Fundação para a Ciência e a Tecnologia (FCT) through national funds (UID/FIS/04434/2013) and Investigador FCT contract IF/01654/2014/CP1215/CT0003., and by FEDER through COMPETE2020 (POCI-01-0145-FEDER-007672). Funding for the Sloan Digital Sky Survey IV has been provided by the Alfred P. Sloan Foundation, the U.S. Department of Energy Office of Science, and the Participating Institutions. SDSS-IV acknowledges support and resources from the Center for High-Performance Computing at the University of Utah. The SDSS web site is www.sdss.org. SDSS-IV is managed by the Astrophysical Research Consortium for the Participating Institutions of the SDSS Collaboration including the Brazilian Participation Group, the Carnegie Institution for Science, Carnegie Mellon University, the Chilean Participation Group, the French Participation Group, Harvard-Smithsonian Center for Astrophysics, Instituto de Astrofísica de Canarias, The Johns Hopkins University, Kavli Institute for the Physics and Mathematics of the Universe (IPMU) / University of Tokyo, the Korean 
Participation Group, Lawrence Berkeley National Laboratory, Leibniz Institut für Astrophysik Potsdam (AIP), MaxPlanck-Institut für Astronomie (MPIA Heidelberg), MaxPlanck-Institut für Astrophysik (MPA Garching), MaxPlanck-Institut für Extraterrestrische Physik (MPE), National Astronomical Observatories of China, New Mexico State University, New York University, University of Notre Dame, Observatário Nacional / MCTI, The Ohio State University, Pennsylvania State University, Shanghai Astronomical Observatory, United Kingdom Participation Group, Universidad Nacional Autónoma de México, University of Arizona, University of Colorado Boulder, University of Oxford, University of Portsmouth, University of Utah, University of Virginia, University of Washington, University of Wisconsin, Vanderbilt University, and Yale University.

\section{REFERENCES}

Abolfathi B., et al., 2018, ApJS, 235, 42

Aguado D. S., et al., 2019, The Astrophysical Journal Supplement Series, 240, 23

Baldry I. K., Glazebrook K., Brinkmann J., Ivezić Ž., Lupton R. H., Nichol R. C., Szalay A. S., 2004, ApJ, 600, 681

Baldwin J. A., Phillips M. M., Terlevich R., 1981, PASP, 93, 5

Balogh M. L., Baldry I. K., Nichol R., Miller C., Bower R., Glazebrook K., 2004, ApJ, 615, L101

Balogh M. L., et al., 2011, MNRAS, 412, 2303

Belfiore F., et al., 2016, MNRAS, 461, 3111

Belfiore F., et al., 2017a, MNRAS, 466, 2570

Belfiore F., et al., 2017b, MNRAS, 469, 151

Belfiore F., et al., 2018, MNRAS, 477, 3014

Bell E. F., et al., 2004, ApJ, 608, 752

Bell E. F., et al., 2012, ApJ, 753, 167

Blanton M. R., 2006, ApJ, 648, 268

Blanton M. R., et al., 2003, ApJ, 594, 186

Blanton M. R., Kazin E., Muna D., Weaver B. A., Price-Whelan A., 2011, AJ, 142, 31

Blanton M. R., et al., 2017, AJ, 154, 28

Block D. L., Wainscoat R. J., 1991, Nature, 353, 48

Block D. L., Puerari I., Knapen J. H., Elmegreen B. G., Buta R., Stedman S., Elmegreen D. M., 2001, A\&A, 375, 761

Boissier S., Prantzos N., 1999, MNRAS, 307, 857

Bolzonella M., et al., 2010, A\&A, 524, A76

Brammer G. B., et al., 2009, ApJ, 706, L173

Bundy K., et al., 2006, ApJ, 651, 120

Bundy K., et al., 2015, ApJ, 798, 7

Calzetti D., Armus L., Bohlin R. C., Kinney A. L., Koornneef J., Storchi-Bergmann T., 2000, ApJ, 533, 682

Cappellari M., Copin Y., 2003, MNRAS, 342, 345

Cappellari M., Emsellem E., 2004, PASP, 116, 138

Cardelli J. A., Clayton G. C., Mathis J. S., 1989, ApJ, 345, 245

Carpineti A., Kaviraj S., Darg D., Lintott C., Schawinski K., Shabala S., 2012, MNRAS, 420, 2139

Cassata P., et al., 2008, A\&A, 483, L39

Chen Y.-M., et al., 2019, arXiv e-prints, p. arXiv:1909.01658

Chiappini C., Matteucci F., Romano D., 2001, ApJ, 554, 1044

Cicone C., et al., 2014, A\&A, 562, A21

Cimatti A., et al., 2013, ApJ, 779, L13

Cirasuolo M., et al., 2007, MNRAS, 380, 585

Citro A., Pozzetti L., Quai S., Moresco M., Vallini L., Cimatti A., 2017, MNRAS, 469, 3108

Couch W. J., Sharples R. M., 1987, MNRAS, 229, 423

Cucciati O., et al., 2006, A\&A, 458, 39

Curti M., Cresci G., Mannucci F., Marconi A., Maiolino R., Esposito S., 2017, MNRAS, 465, 1384
Dasyra K. M., et al., 2006, ApJ, 651, 835

De Lucia G., Springel V., White S. D. M., Croton D., Kauffmann G., 2006, MNRAS, 366, 499

Dopita M. A., Sutherland R. S., 2003, Astrophysics of the diffuse universe

Dopita M. A., Kewley L. J., Heisler C. A., Sutherland R. S., 2000, ApJ, 542, 224

Dopita M. A., et al., 2006, ApJS, 167, 177

Dressler A., Gunn J. E., 1983, ApJ, 270, 7

Ellison S. L., Sánchez S. F., Ibarra-Medel H., Antonio B., Mendel J. T., Barrera-Ballesteros J., 2018, MNRAS, 474, 2039

Eskridge P. B., et al., 2002, ApJS, 143, 73

Faber S. M., et al., 2007, ApJ, 665, 265

Fabian A. C., 2012, ARA\&A, 50, 455

Fall S. M., Efstathiou G., 1980, MNRAS, 193, 189

García-Benito R., et al., 2015, A\&A, 576, A135

Genzel R., Tacconi L. J., Rigopoulou D., Lutz D., Tecza M., 2001, ApJ, 563, 527

Goddard D., et al., 2017a, MNRAS, 465, 688

Goddard D., et al., 2017b, MNRAS, 466, 4731

Gogarten S. M., et al., 2010, ApJ, 712, 858

González Delgado R. M., et al., 2014, A\&A, 562, A47

González Delgado R. M., et al., 2015, A\&A, 581, A103

González Delgado R. M., et al., 2016, A\&A, 590, A44

Goto T., Yamauchi C., Fujita Y., Okamura S., Sekiguchi M., Smail I., Bernardi M., Gomez P. L., 2003, MNRAS, 346, 601

Hibbard J. E., van Gorkom J. H., 1996, AJ, 111, 655

Ho I.-T., Kudritzki R.-P., Kewley L. J., Zahid H. J., Dopita M. A., Bresolin F., Rupke D. S. N., 2015, MNRAS, 448, 2030

Hogg D. W., et al., 2003, ApJ, 585, L5

Huertas-Company M., Aguerri J. A. L., Bernardi M., Mei S., Sánchez Almeida J., 2011, A\&A, 525, A157

Hummer D. G., Storey P. J., 1987, MNRAS, 224, 801

Ilbert O., et al., 2013, A\&A, 556, A55

Kashino D., Renzini A., Silverman J. D., Daddi E., 2016, ApJ, 823, L24

Kauffmann G., et al., 2003, MNRAS, 346, 1055

Kaviraj S., 2010, MNRAS, 408, 170

Kennicutt Jr. R. C., 1998, ARA\&A, 36, 189

Kennicutt R. C., Evans N. J., 2012, ARA\&A, 50, 531

Kewley L. J., Dopita M. A., 2002, ApJS, 142, 35

Kewley L. J., Dopita M. A., Sutherland R. S., Heisler C. A., Trevena J., 2001, ApJ, 556, 121

Kewley L. J., Dopita M. A., Leitherer C., Davé R., Yuan T., Allen M., Groves B., Sutherland R., 2013, ApJ, 774, 100

Kormendy J., Kennicutt Jr. R. C., 2004, ARA\&A, 42, 603

Kriek M., van der Wel A., van Dokkum P. G., Franx M., Illingworth G. D., 2008, ApJ, 682, 896

Kroupa P., 2001, MNRAS, 322, 231

Larson R. B., 1976, MNRAS, 176, 31

Laurikainen E., Salo H., 2002, MNRAS, 337, 1118

Law D. R., et al., 2016, AJ, 152, 83

Le Borgne D., et al., 2006, ApJ, 642, 48

Lee J. C., et al., 2009, ApJ, 706, 599

Levesque E. M., Kewley L. J., Larson K. L., 2010, AJ, 139, 712

Lin L., et al., 2019, ApJ, 872, 50

Martin D. C., et al., 2005, ApJ, 619, L1

Martin D. C., et al., 2007, ApJS, 173, 342

Matthee J., Schaye J., 2019, MNRAS, 484, 915

Mendel J. T., Simard L., Ellison S. L., Patton D. R., 2013, MNRAS, 429, 2212

Mok A., et al., 2013, MNRAS, 431, 1090

Morselli L., Popesso P., Cibinel A., Oesch P. A., Montes M., Atek H., Illingworth G. D., Holden B., 2018, arXiv e-prints,

Mortlock A., Conselice C. J., Bluck A. F. L., Bauer A. E., Grützbauch R., Buitrago F., Ownsworth J., 2011, MNRAS, 413,2845

Moustakas J., et al., 2013, ApJ, 767, 50 
Muñoz-Mateos J. C., Boissier S., Gil de Paz A., Zamorano J., Kennicutt Jr. R. C., Moustakas J., Prantzos N., Gallego J., 2011, ApJ, 731, 10

Muzzin A., et al., 2012, ApJ, 746, 188

Muzzin A., et al., 2013, ApJS, 206, 8

Nagao T., Maiolino R., Marconi A., 2006, A\&A, 459, 85

Osterbrock D. E., 1989, Astrophysics of gaseous nebulae and active galactic nuclei

Paalvast M., Brinchmann J., 2017, MNRAS, 470, 1612

Peng Y.-j., et al., 2010, ApJ, 721, 193

Pezzulli G., Fraternali F., 2016, MNRAS, 455, 2308

Pezzulli G., Fraternali F., Boissier S., Muñoz-Mateos J. C., 2015, MNRAS, 451, 2324

Pichon C., Pogosyan D., Kimm T., Slyz A., Devriendt J., Dubois Y., 2011, MNRAS, 418, 2493

Poggianti B. M., Bridges T. J., Komiyama Y., Yagi M., Carter D., Mobasher B., Okamura S., Kashikawa N., 2004, ApJ, 601, 197

Poggianti B. M., et al., 2008, ApJ, 684, 888

Pozzetti L., et al., 2010, A\&A, 523, A13

Prantzos N., Boissier S., 2000, MNRAS, 313, 338

Prendergast K. H., 1983, in Athanassoula E., ed., IAU Symposium Vol. 100, Internal Kinematics and Dynamics of Galaxies. pp 215-220

Quai S., Pozzetti L., Citro A., Moresco M., Cimatti A., 2018, MNRAS,

Quintero A. D., et al., 2004, ApJ, 602, 190

Regan M. W., Teuben P., 2003, ApJ, 582, 723

Rosales-Ortega F. F., Díaz A. I., Kennicutt R. C., Sánchez S. F., 2011, MNRAS, 415, 2439

Rothberg B., Joseph R. D., 2004, AJ, 128, 2098

Salim S., 2014, Serbian Astronomical Journal, 189, 1

Salim S., et al., 2007, ApJS, 173, 267

Sánchez-Blázquez P., Rosales-Ortega F., Diaz A., Sánchez S. F., 2014, MNRAS, 437, 1534

Sánchez S. F., et al., 2014, A\&A, 563, A49

Sanders D. B., Soifer B. T., Elias J. H., Madore B. F., Matthews K., Neugebauer G., Scoville N. Z., 1988, ApJ, 325, 74

Schawinski K., et al., 2014, MNRAS, 440, 889

Schiminovich D., et al., 2007, ApJS, 173, 315

Schweizer F., Seitzer P., 1992, AJ, 104, 1039

Sellwood J. A., Wilkinson A., 1993, Reports on Progress in Physics, 56, 173

Spindler A., et al., 2018, MNRAS, 476, 580

Stewart K. R., Brooks A. M., Bullock J. S., Maller A. H., Diemand J., Wadsley J., Moustakas L. A., 2013, ApJ, 769, 74

Strateva I., et al., 2001, AJ, 122, 1861

Tacchella S., et al., 2015, Science, 348, 314

Tacchella S., Dekel A., Carollo C. M., Ceverino D., DeGraf C., Lapiner S., Mandelker N., Primack Joel R., 2016, MNRAS, 457,2790

Tacconi L. J., et al., 2013, ApJ, 768, 74

Tal T., van Dokkum P. G., Nelan J., Bezanson R., 2009, AJ, 138, 1417

Tinker J. L., Wechsler R. H., Zheng Z., 2010, ApJ, 709, 67

Vazdekis A., Ricciardelli E., Cenarro A. J., Rivero-González J. G., Díaz-García L. A., Falcón-Barroso J., 2012, MNRAS, 424, 157

Wang J., et al., 2011, Monthly Notices of the Royal Astronomical Society, 412, 1081

Wang E., Lilly S. J., Pezzulli G., Matthee J., 2019, arXiv e-prints,

Wild V., Walcher C. J., Johansson P. H., Tresse L., Charlot S., Pollo A., Le Fèvre O., de Ravel L., 2009, MNRAS, 395, 144

Wild V., Almaini O., Dunlop J., Simpson C., Rowlands K., Bowler R., Maltby D., McLure R., 2016, MNRAS, 463, 832

Williams R. J., Quadri R. F., Franx M., van Dokkum P., Labbé I., 2009, ApJ, 691, 1879

Willmer C. N. A., et al., 2006, ApJ, 647, 853
Wu P.-F., Gal R. R., Lemaux B. C., Kocevski D. D., Lubin L. M., Rumbaugh N., Squires G. K., 2014, ApJ, 792, 16

Wyder T. K., et al., 2007, ApJS, 173, 293

Zabludoff A. I., Zaritsky D., Lin H., Tucker D., Hashimoto Y., Shectman S. A., Oemler A., Kirshner R. P., 1996, ApJ, 466, 104

Zaritsky D., Kennicutt Jr. R. C., Huchra J. P., 1994, ApJ, 420, 87 


\section{ON-LINE MATERIAL A: MAIN PARAMETERS RADIAL PROFILES OF INDIVIDUAL GALAXIES}

In this section we show the radial profiles for each galaxy in the QGs and SFs samples of the main parameters analysed in this paper (i.e. $[\mathrm{O} \mathrm{III}] / \mathrm{H} \alpha,[\mathrm{N} \mathrm{II}] /[\mathrm{O} \mathrm{II}]$, ionisation parameter $\log (\mathrm{U})$, gas-phase metallicity $\mathrm{Z}$ and the SFR surface density $\Sigma \mathrm{SFR}$ ).

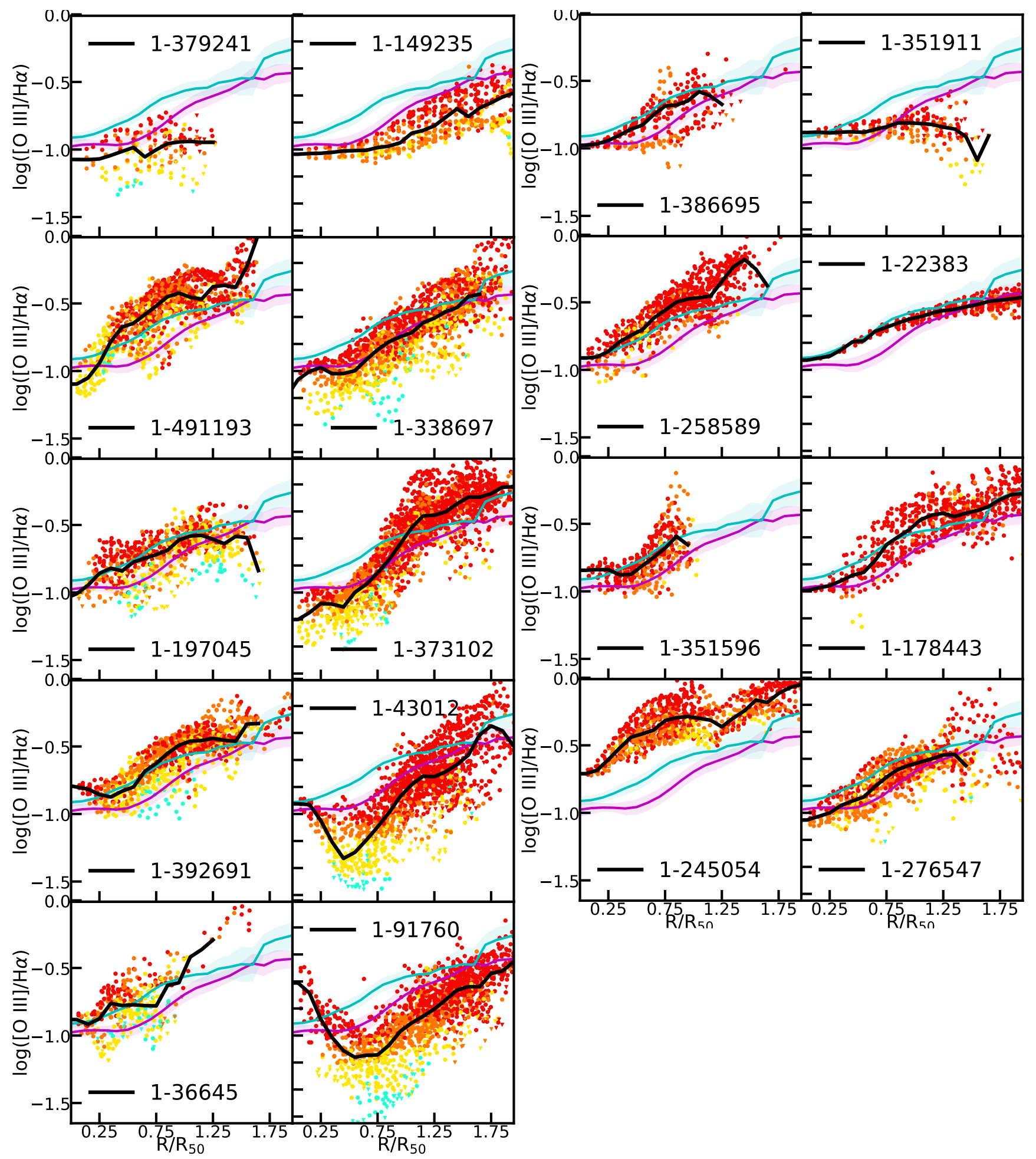

Figure A1. Radial profiles of $[\mathrm{O} \mathrm{III}] / \mathrm{H} \alpha$ ratio of each galaxy belonging to QG sample (left) and to SF sample (right). The black curves represent the median of the relations in bins of width $0.1 \mathrm{R} / \mathrm{R}_{50}$, while the blue ones represent the $16-84^{\text {th }}$ percentile of the relations. Each round dot represents a spaxel in which the $\mathrm{S} / \mathrm{N}\left(\left[\mathrm{O}_{\mathrm{III}}\right]\right) \geq 2$, while the square dots represent spaxels in which the $\mathrm{S} / \mathrm{N}\left(\left[\mathrm{O}_{\mathrm{III}}\right]\right)<2$ and their $[\mathrm{O} I I I] / \mathrm{H} \alpha$ values are upper-limits. The dots colour code is the same as in Figure 7 , and it is based on the position of each spaxel on the $[\mathrm{O}$ III $] / \mathrm{H} \alpha$ vs $[\mathrm{N} \mathrm{II}] /[\mathrm{O}$ II] diagram (Figure 1 and Figure 6). The cyan is representing quenching regions, followed by the yellow for the galactic regions that lie between $3 \times 1 \sigma$ and $1 \sigma$ of the diagram, orange for those between $1 \sigma$ and the median and red for regions of pure star-formation that are above the median of the diagram. The magenta and cyan curves represent the average radial profiles of QG and SFs populations (as in Figure 11).

MNRAS 000, 1-?? (2019) 


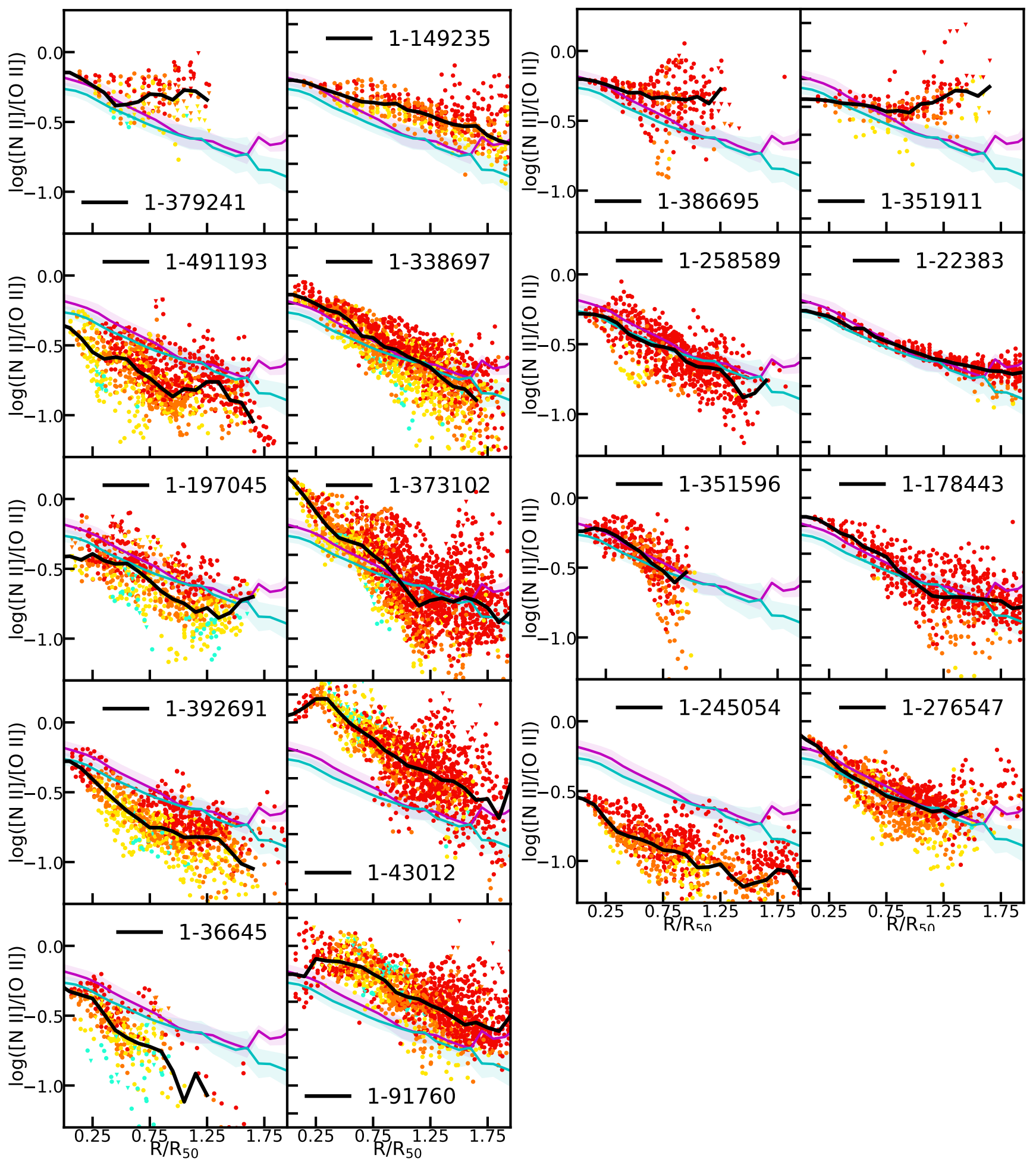

Figure A2. Radial profiles of $\left[\mathrm{N}_{\mathrm{II}}\right] /\left[\mathrm{O}_{\mathrm{II}}\right]$ of each galaxy belonging to QG sample (left) and to SF sample (right). See Figure A1 for panels description. 


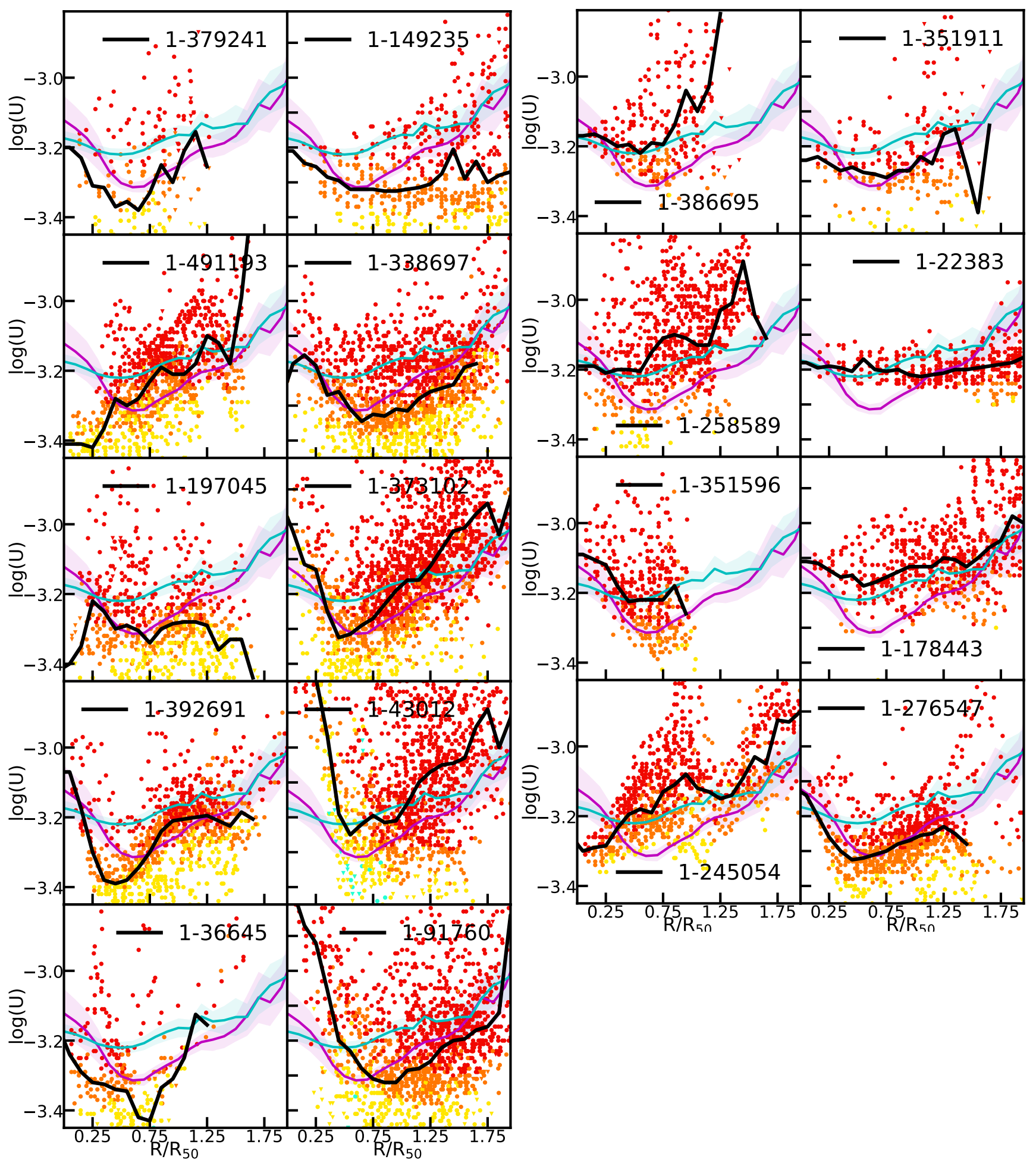

Figure A3. Radial profiles of ionisation parameter $\log (\mathrm{U})$ of each galaxy belonging to QG sample (left) and to SF sample (right). See Figure A1 for panels description. 

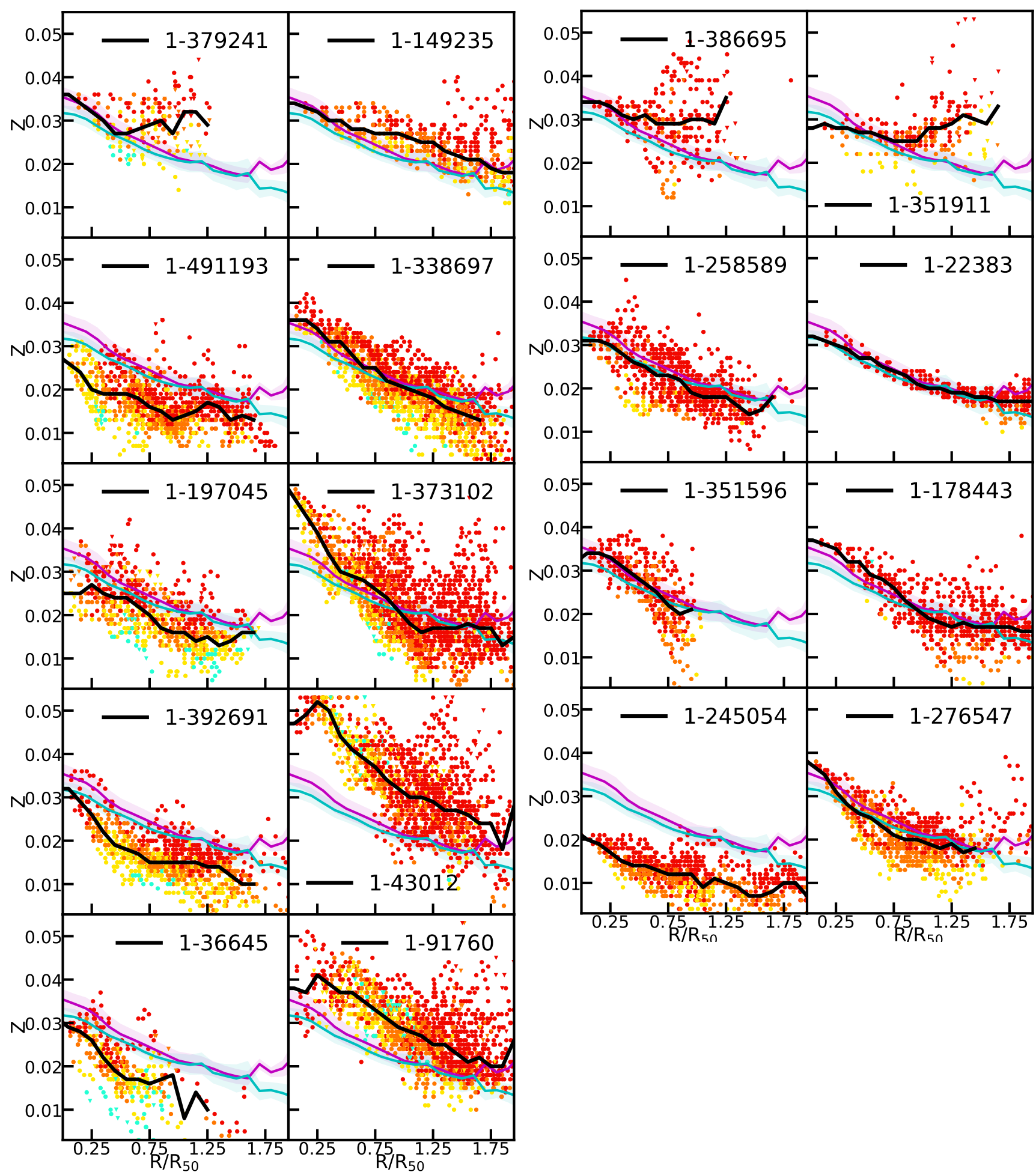

Figure A4. Radial profiles of gas phase metallicity $\mathrm{Z}$ of each galaxy belonging to QG sample (left) and to SF sample (right). See Figure A1 for panels description. 

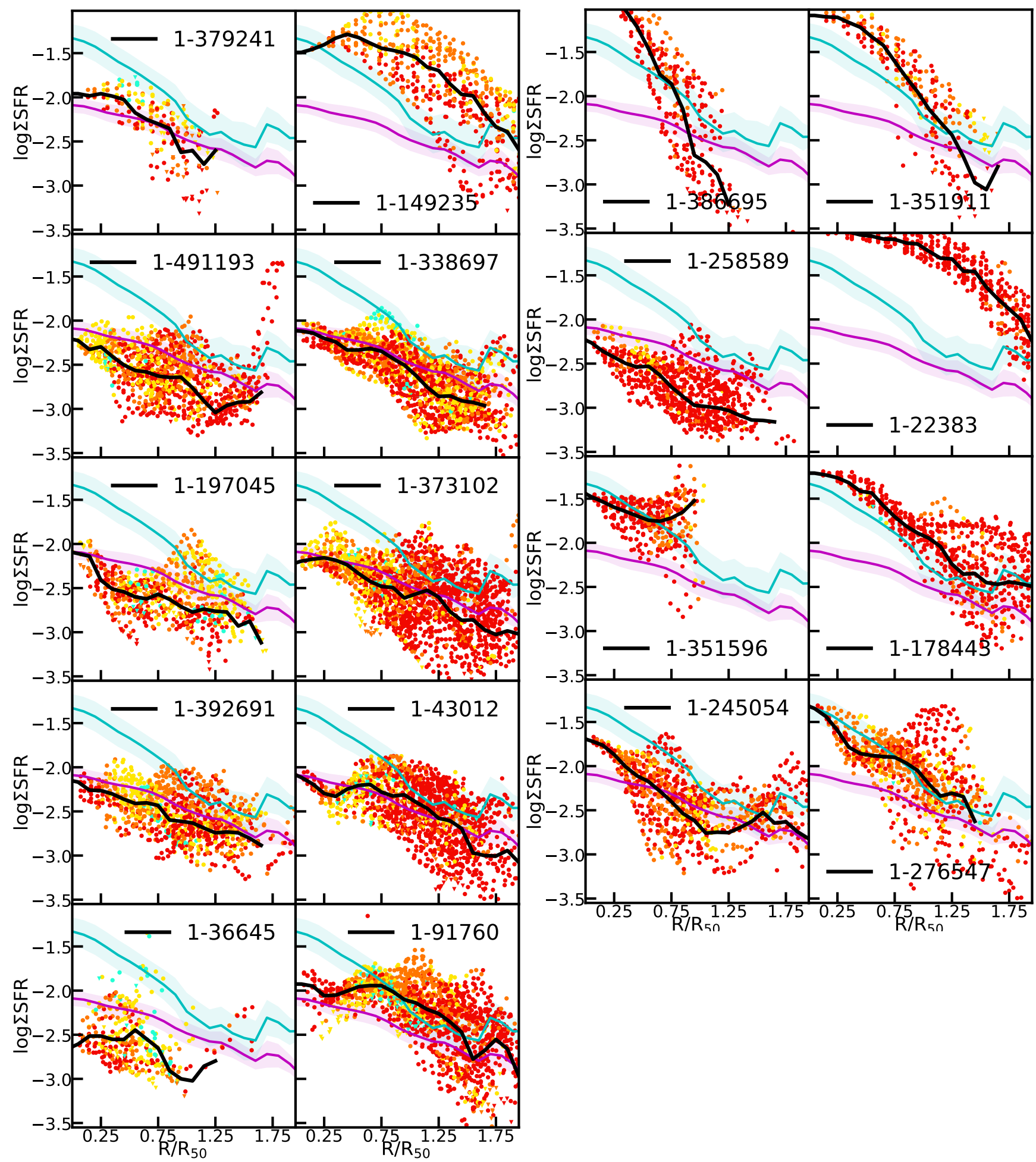

Figure A5. Radial profiles of the star formation rate surface density $\Sigma$ SFR of each galaxy belonging to QG sample (left) and to SF sample (right). See Figure A1 for panels description. 